\title{
Iron-TAML Complexes: A Computational Approach to Improving Stability
}

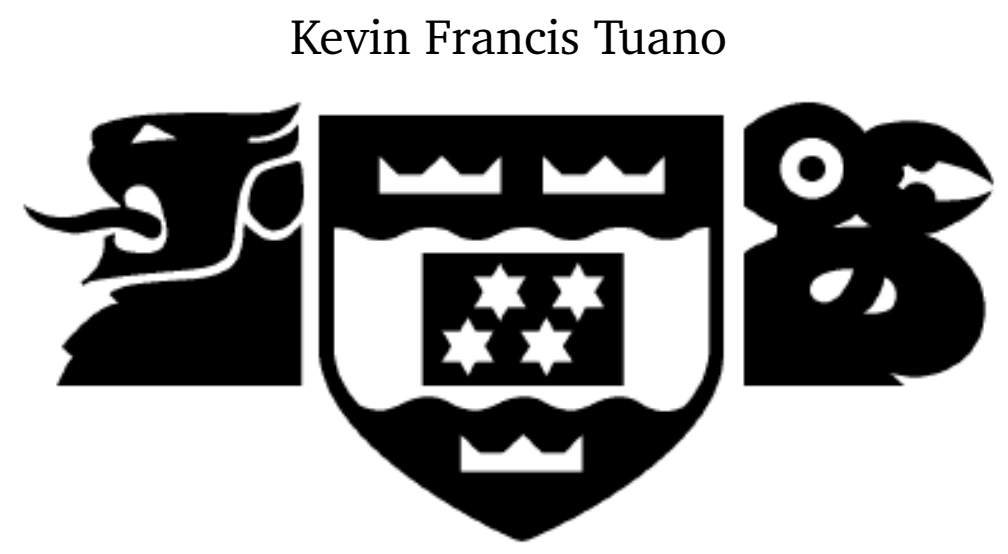

School of Chemical and Physical Sciences

Victoria University of Wellington

2016

A thesis submitted to Victoria University of Wellington in partial fulfilment for the degree of Master of Science in Chemistry 


\section{Abstract}

Researchers at the Institute for Green Oxidation Chemistry of the Carnegie Mellon University developed a group of catalysts called tetra amido macrocyclic ligand (TAML) activators. The purpose of that research was that TAML activators would breakdown pollutants in the presence of a sacrificial oxidant. Furthermore, the catalyst was designed to decompose on a required timescale, as to not damage the environment by prolonged exposure. Since the initial designs from the 1980's, the TAML structure has undergone significant changes to increase efficiency or selectivity. Other uses of this group of catalysts have been explored, namely, the oxidation of water to molecular oxygen.

This work presents a computational study using Density Functional Theory (DFT) which addresses the issue regarding the stability of certain iron-TAML intermediates in the water oxidation mechanism. Hence, the work seeks to explore how changing certain groups on the TAML ring can affect the stability of the reactive intermediates and the activation energy of the nucleophilic attack within the mechanism. The work highlights the importance of the fluorinated tail of the TAML structure in the accessibility of the desired transition state. 


\section{Acknowledgements}

The decision to embark on a journey of further study was one take up in a pursuit of a great challenge. Indeed it was a great challenge and yet a fulfilling journey accomplished only through the guidance of special people. This page is dedicated to express my appreciation for those groups and individuals who have been a great help and source of encouragement throughout my time as Masters student at Victoria University of Wellington.

Foremost, I would like to extend my deep gratitude toward my supervisor Dr Matthias Lein. It was his undergraduate lectures (at the time, and some are still, difficult to grasp) that evoked my curiosity in Computational Chemistry. He has been an exceptional source of guidance and support. His enthusiasm for the work and relaxed nature have been essential in creating a great group environment.

My family has been very supportive of my decision for further study. I am inclined to thank them for listening to my complaints and struggles but also rejoice in successes. I would also like to thank my friends and peers (Wong, Nina, and Muhammad) who have impelled me to strive for the best.

Furthermore, I would like to acknowledge the School of Chemical and Physical Science at Victoria University of Wellington for the Curtis-Gordon Research Scholarship in Chemistry that has offset the cost of my study. Computing resources were provided by Victoria University of Wellington High Performance Computing Facilities Heisenberg and ScifacHPC. 


\section{Contents}

$\begin{array}{ll}\text { Abstract } & \text { i }\end{array}$

Acknowledgements ii

List of Figures vi vi

List of Tables $\quad$ xii

1 Introduction $\quad 1$

1.1 Structure of TAML Activators . . . . . . . . . . 2

1.2 Early Designs and the Development Process . . . . . . . . 3

1.3 Applications of TAML Activators in Green Chemistry . . . . . 5

1.3.1 Pulp and Paper Industry $\ldots \ldots \ldots$

1.3.2 Textile Industry $\ldots \ldots \ldots \ldots$

1.3.3 Military Applications . . . . . . . . . . 7

1.3.4 Desulfurisation of Fuels . . . . . . . . . . . 7 
1.3.5 Pesticide and Pharmaceuticals . . . . . . . . . 8

1.3.6 Fast Water Oxidation . . . . . . . . . . 9

1.4 Mechanistic Studies . . . . . . . . . . . . . 9

2 Methodology 12

2.1 Preamble ........................ 12

2.1.1 Wavefunction-based Methods ........... 13

2.1.2 Density Functional Theory . . . . . . . . . . 15

2.2 Transition State Theory $\ldots \ldots \ldots 17$

2.3 Computational Methods ............... 19

$\begin{array}{llr}3 & \text { Results } & 20\end{array}$

3.1 Mechanism Overview . . . . . . . . . . . . . . 21

3.2 Proton-Coupled Electron Transfer Steps . . . . . . . . . . 23

3.3 Transition State: Water Nucleophilic Attack . . . . . . . . 26

3.4 Oxygen Generation . . . . . . . . . . . . . . 30

4 Discussion $\quad 36$

4.1 Comparison of Results . . . . . . . . . . . . . 36

4.2 Stability of Reactive Intermediates $\ldots . . \ldots 37$

4.3 Limiting Factors of Ligand Design . . . . . . . . . . . . . 39 
CONTENTS

4.4 Thermodynamics of Reactions . . . . . . . . . . . . . . . . 41

5 Conclusion

Appendix A

Appendix B

Appendix C

48

Bibliography

54 


\section{List of Figures}

1.1 General TAML structure that will be discussed. Other variations for the macrocycle do exist and will be mentioned in subsequent sections . . . . . . . . . . . . . . . . 2

1.2 The iterative design process of the TAML macrocycle. Reproduced with permission; from Collins [2] copyright (C) 2002 American Chemical Society. . . . . . . . . . . . . . . . . . . . 3

1.3 Design loop used by Collins to obtain oxidation resistant ligands. Reproduced with permission; from Collins [2] copyright (c) 2002 American Chemical Society. . . . . . . . . . . . . . 4

1.4 Discolouration example. Middle and right vials contain textile dyes, with Fe-TAML added to vial on right. Vial on left contains water for reference. Reproduced from Environmental Health Perspectives $[6] \ldots \ldots \ldots \ldots$. . . . . . . . . . . . 6

1.5 Example of pollutant degradation for fenitrothion. Reproduced with permission; from Collins [28] copyright (c) 2006 American Chemical Society. . . . . . . . . . . . . . . . 8 
1.6 Proposed fast water oxidation pathway. Red structures display the proton coupled electron transfer (PCET) pathway along with the water nucleophilic attack and oxygen generation. TAML rings omitted for clarity. . . . . . . . . . . . . . . 10

1.7 Local solvation shell proposed by Cramer. Reproduced from [32] with permission from the PCCP Owner Societies . . . . . 10

2.1 Classical representation of a chemical reaction. Reaction proceeds along intrinsic reaction coordinate (IRC). . . . . . 17

2.2 Saddle point for illustrative purposes. . . . . . . . . . . . 18

3.1 TAML structure substitution arrangements. $\mathbf{A}$ is an experimental structure. B is a proposed structure with full electronwithdrawing group (EWG) substitution. $\mathbf{C}$ is a proposed structure with mixture of EWG's and electron-donating groups (EDG). D is a variant structure with mixture of EWG's and electrondonating groups (EDG). $\mathrm{E}$ is the final proposed structure with full electron-donating group (EDG) substitution arrangement.

3.2 General fast water oxidation reaction scheme. Structures for each step labelled below in bold. PCET steps separated into individual oxidation and proton loss steps for resolution of the

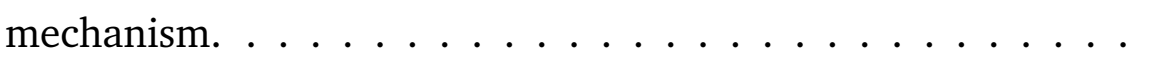

3.3 PCET steps of mechanism for experimental structure A. Relative energies with respect to starting materials given below structure while energetic differences between steps in the mechanism are given on dotted lines between structure. Calculated at PBE0/Def2-TZVP level of theory. All energies in $\mathrm{kcal}^{\mathrm{mol}}{ }^{-1}$. 
3.4 PCET steps of mechanism for proposed structures $\mathbf{D}$ and E. Relative energies with respect to starting materials given below structure while energetic differences between steps in the mechanism are given on dotted lines between structure. Calculated at PBE0/Def2-TZVP level of theory. All energies in $\mathrm{kcal} \mathrm{mol}^{-1}$. For a more comprehensive list of relative energies refer to Tables 3.1 and $3.2 \ldots \ldots \ldots \ldots$

3.5 Nucleophilic attack of water on the reactive intermediate 6A. Four water molecule local solvation shell present to facilitate proton transfers and solvation of the latter hydronium ion. Relative energies with respect to starting materials given below structure while energetic differences between steps in the mechanism are given on dotted lines between structure. Calculated at PBE0/Def2-TZVP level of theory. All energies in kcal $\mathrm{mol}^{-1}$. . . . . . . . . . . . . .

3.6 Nucleophilic attack of water on the reactive intermediate 6B. Four water molecule local solvation shell present to facilitate proton transfers and solvation of the latter hydronium ion. Relative energies with respect to starting materials given below structure while energetic differences between steps in the mechanism are given on dotted lines between structure. Calculated at PBE0/Def2-TZVP level of theory. All energies in kcal $\mathrm{mol}^{-1}$. . . . . . . . . . . . . . . 28 
3.7 Nucleophilic attack of water on the reactive intermediate 6D. Four water molecule local solvation shell present to facilitate proton transfers and solvation of the latter hydronium ion. Relative energies with respect to starting materials given below structure while energetic differences between steps in the mechanism are given on dotted lines between structure. Calculated at PBE0/Def2-TZVP level of theory. All energies in $\mathrm{kcal} \mathrm{mol}^{-1}$. . . . . . . . . . . . . .

3.8 Nucleophilic attack of water on the reactive intermediate 6C. Four water molecule local solvation shell present to facilitate proton transfers and solvation of the latter hydronium ion. Relative energies with respect to starting materials given below structure while energetic differences between steps in the mechanism are given on dotted lines between structure. Calculated at PBE0/Def2-TZVP level of theory. All energies in kcal mol ${ }^{-1} \ldots \ldots \ldots \ldots \ldots$

3.9 Oxygen generation steps of the mechanism for experimental structure A. Relative energies with respect to starting materials given below structure while energetic differences between steps in the mechanism are given on dotted lines between structure. Calculated at PBE0/Def2-TZVP level of theory. All energies in $\mathrm{kcal} \mathrm{mol}^{-1}$. . . . . . . . . . . . .

3.10 Oxygen generation steps of the mechanism for proposed structure B. Relative energies with respect to starting materials given below structure while energetic differences between steps in the mechanism are given on dotted lines between structure. Calculated at PBE0/Def2-TZVP level of theory. All energies in $\mathrm{kcal} \mathrm{mol}^{-1}$. For a more comprehensive list of relative energies refer to Tables 3.1 and $3.2 \ldots \ldots \ldots \ldots \ldots$ 
4.1 MO contour plots of singly occupied molecular orbitals (SOMOs) for reactive intermediate structure at PBE0/Def2-TZVP level of theory. Molecules rotated to give clarity for the orbital

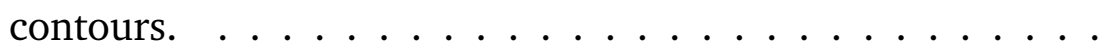

4.2 Space-filling model of a structure along the optimisation of TS E that had an imaginary frequency which did not correspond the nucleophilic attack. Structures $\mathbf{7}$ and $\mathbf{8}$ provide insight that the solvation shell is not likely to facilitate the desired

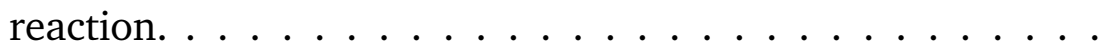

B1 MO contour plots of singly occupied molecular orbitals (SOMOs) for reactive intermediate structure at PBE0/Def2-TZVP level of theory. Molecules rotated to give clarity for the orbital

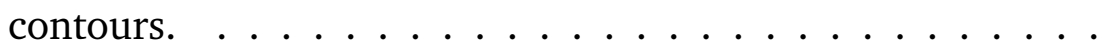

C2 Full mechanism of experimental structure A. Molecule rotated for structures 7 and TS for clarity of local solvation shell. Relative energies with respect to starting materials given below structure while energetic differences between steps in the mechanism are given on dotted lines between structure. Calculated at PBE0/Def2-TZVP level of theory. All energies in $\mathrm{kcal} \mathrm{mol}^{-1} \ldots \ldots \ldots \ldots \ldots \ldots \ldots$

C3 Full mechanism of proposed structure B. Molecule rotated for structures 7 and TS for clarity of local solvation shell. Relative energies with respect to starting materials given below structure while energetic differences between steps in the mechanism are given on dotted lines between structure. Calculated at PBE0/Def2-TZVP level of theory. All energies in $\mathrm{kcal} \mathrm{mol}^{-1}$ 
C4 Full mechanism of proposed structure C. Relative energies with respect to starting materials given below structure while energetic differences between steps in the mechanism are given on dotted lines between structure. Calculated at PBE0/Def2TZVP level of theory. All energies in $\mathrm{kcal}^{\mathrm{mol}}{ }^{-1} \ldots \ldots$

C5 Full mechanism of proposed structure D. Molecule rotated for structures 7 and TS for clarity of local solvation shell. Relative energies with respect to starting materials given below structure while energetic differences between steps in the mechanism are given on dotted lines between structure. Calculated at PBE0/Def2-TZVP level of theory. All energies in $\mathrm{kcal} \mathrm{mol}^{-1}$

C6 Full mechanism of proposed structure E (TS omitted). Molecule rotated for structure $\mathbf{7}$ for clarity of local solvation shell. Relative energies with respect to starting materials given below structure while energetic differences between steps in the mechanism are given on dotted lines between structure. Calculated at PBE0/Def2-TZVP level of theory. All energies in $\mathrm{kcal} \mathrm{mol}^{-1}$ 


\section{List of Tables}

3.1 Relative energies with respect to previous structure. Calculated at PBE0/Def2-TZVP level of theory. All energies in kcal $\mathrm{mol}^{-1} \ldots \ldots \ldots \ldots \ldots \ldots \ldots$

3.2 Relative energies with respect to starting material. Calculated at PBE0/Def2-TZVP level of theory. All energies in $\mathrm{kcal} \mathrm{mol}^{-1}$.

4.1 Relative energies of experimental structure $\mathbf{A}$ with respect to starting material. Comparing PBE0/Def2-TZVP results with those of Cramer (M06L/6-31+G(d) with ECP10MDF SDD pseudopotential on Fe). [32]. All energies in $\mathrm{kcal} \mathrm{mol}^{-1}$. . . . . . . 37

4.2 Wiberg bond indices from NBO calculation at PBE0/Def2-TZVP level of theory. . . . . . . . . . . . . . . . . . . 4 41

4.3 Relative Enthalpies and Gibb's free energies for structures TS A, TS B, TS C and TS D. Calculated at PBE0/Def2-TZVP level of theory. All energies in $\mathrm{kcal} \mathrm{mol}^{-1}$. . . . . . . . . . . 4 42

A1 Sum of electronic and zero-point energies in a.u. calculated at PBE0/Def2-TZVP level of theory. . . . . . . . . . . . . . 45

A2 Sum of electronic and thermal energies in a.u. calculated at PBE0/Def2-TZVP level of theory. . . . . . . . . . . . . . . 46 
A3 Sum of electronic and thermal free energies in a.u. calculated at PBE0/Def2-TZVP level of theory. . . . . . . . . . . . 46 


\section{Chapter 1}

\section{Introduction}

Chemical contamination is an increasing problem around the world due to industrial and agricultural expansion. Though much effort is placed on fixing the processes which contribute to such contamination, treatment of the affected areas is a more immediate concern. Green Chemistry can attenuate the damage caused by traditional chemistry on the environment. [1]

An aspect of green chemistry, green oxidation, focuses on treatment by mimicking natural processes. The biggest challenges faced in this area of chemistry is to develop new technologies for: (a) conversion of solar energy to either electrical or chemical energy (b) Pollution clean up and (c) Renewable chemical feedstocks. [2, 3] The overall goal is to promote sustainability.

Nature operates differently from industry in the aspect of oxidation chemistry. Contamination by chemicals is a growing problem around the world due to industrial and agricultural proliferation. Dumping waste from mills or farms contribute to most of the chemical pollutants found in rivers and lakes. These include dyes from textile mills, lignin waste and organochlorines from pulp mills, herbicides and pesticides from farms, and general sewage. [1] Thus, by adopting natural oxidants such as hydrogen peroxide and oxygen, the environmental impact of such pollutants can be alleviated. 
Researchers from the Institute for Green Oxidation Chemistry of the Carnegie Mellon University, have developed a class of compounds which catalyse oxidation in the presence of hydrogen peroxide and water called tetra amido macrocylic ligand (TAML) activators. [4]

\subsection{Structure of TAML Activators}

The TAML structure has undergone many changes since the original tetra dentate designs in the 1980's [2, 4, 5] The class of TAML activators which are deemed to be environmentally safe are the iron-TAML complexes. The rationale being, that all the elements within the iron-TAML complex (iron, carbon, nitrogen, oxygen, hydrogen) are naturally abundant, hence, any degradation of the catalyst would be of little environmental concern. [2, 6]<smiles>[X]c1cc2c(cc1[X])N(C(=O)C([R])([R])C)C(=O)C([R])([R])N1CN2C(=O)C([R])([R])C1=O</smiles>

Figure 1.1: General TAML structure that will be discussed. Other variations for the macrocycle do exist and will be mentioned in subsequent sections

The macrocycle coordinates to the iron(III) centre through four nitrogens as shown in Figure 1.1. There is a degree of aromaticity in the macrocycle, to which the structure can be described as loosely based on a porphyrinlike skeleton. Collins and coworkers designed iron-TAML to mimic enzymes which catalyse oxidation, peroxidases and cytochrome P-450 enzymes. $[1,7$, 8] 


\subsection{Early Designs and the Development Process}

Early work by Collins in the 1980's was a ligand design methodology that would produce more effective and robust oxidation catalysts. [2] The tetradentate structure of TAML activators as such is to ensure that there is a coordination site for the oxidant. Moreover, the catalyst must not undergo Fenton chemistry which is especially challenging if the metal centre is iron. [9] Fenton chemistry can be used to oxidise pollutants but was deemed not selective enough as radicals are produced. [2] The metal centre seemed to change with different iterations (manganese, chromium, cobalt to name a few) until iron became the preferred metal for environmental reasons as mentioned previously. $[5,10,11,12,13,14]$

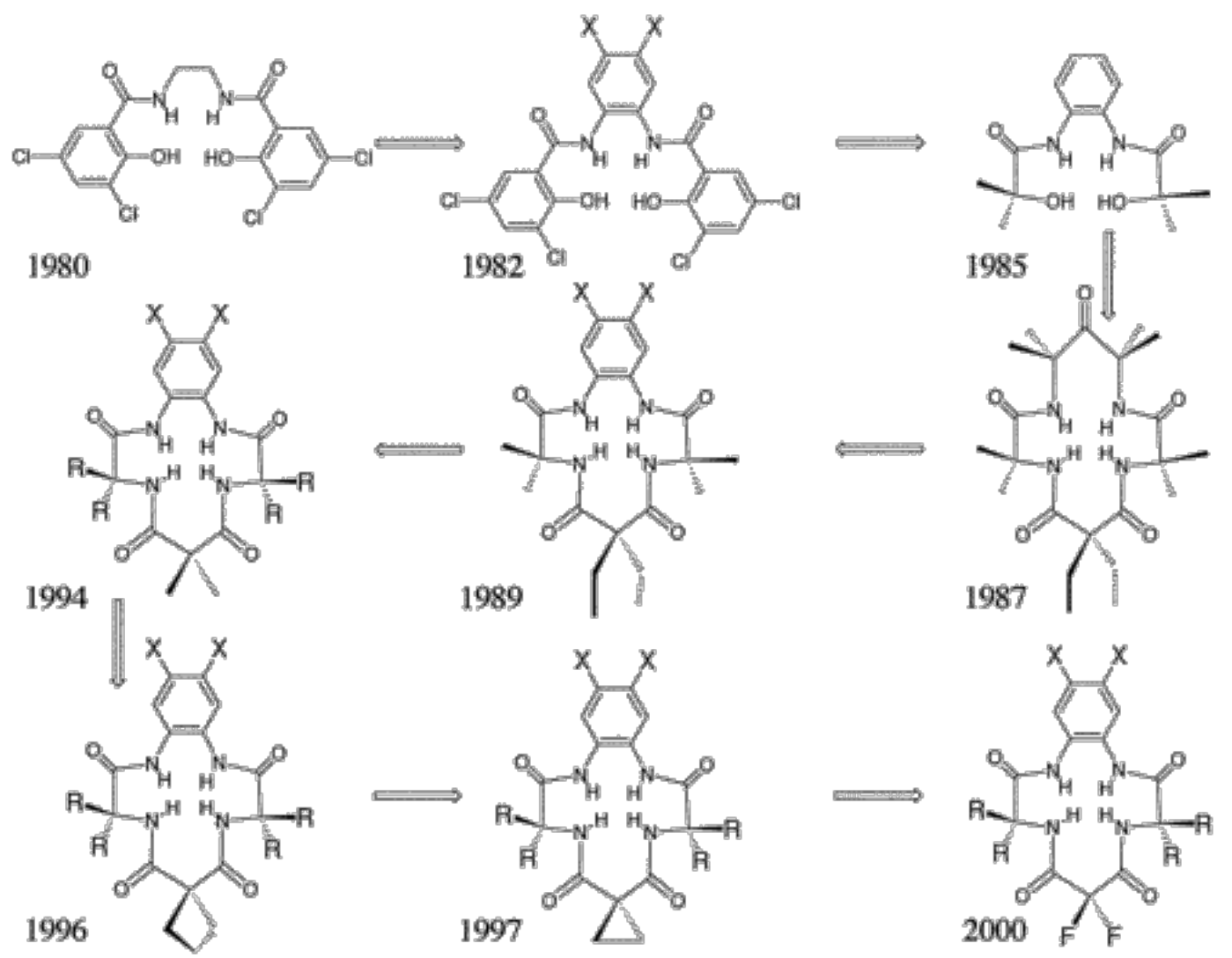

Figure 1.2: The iterative design process of the TAML macrocycle. Reproduced with permission; from Collins [2] copyright (c) 2002 American Chemical Society. 
Iterations (Figure 1.2) are the product of a design process to obtain more resilient catalysts by Collins and coworkers. This involves (a) propose a ligand structure which is oxidation resistant and make the metal complex (b) oxidise the complex until it decays (c) identify vulnerabilities and (d) modify the weak site. This design process (Figure 1.3) was built upon the foundation of ligand protection rules set by Collins and coworkers in the 1994 which enables the TAML ring to decay in a somewhat controlled and known fashion. [2] This approach to ligand design requires rigorous testing in a laboratory, a theoretical approach to the existing systems may be a more efficient way to understanding the next step to the TAML design and complement experimental efforts.

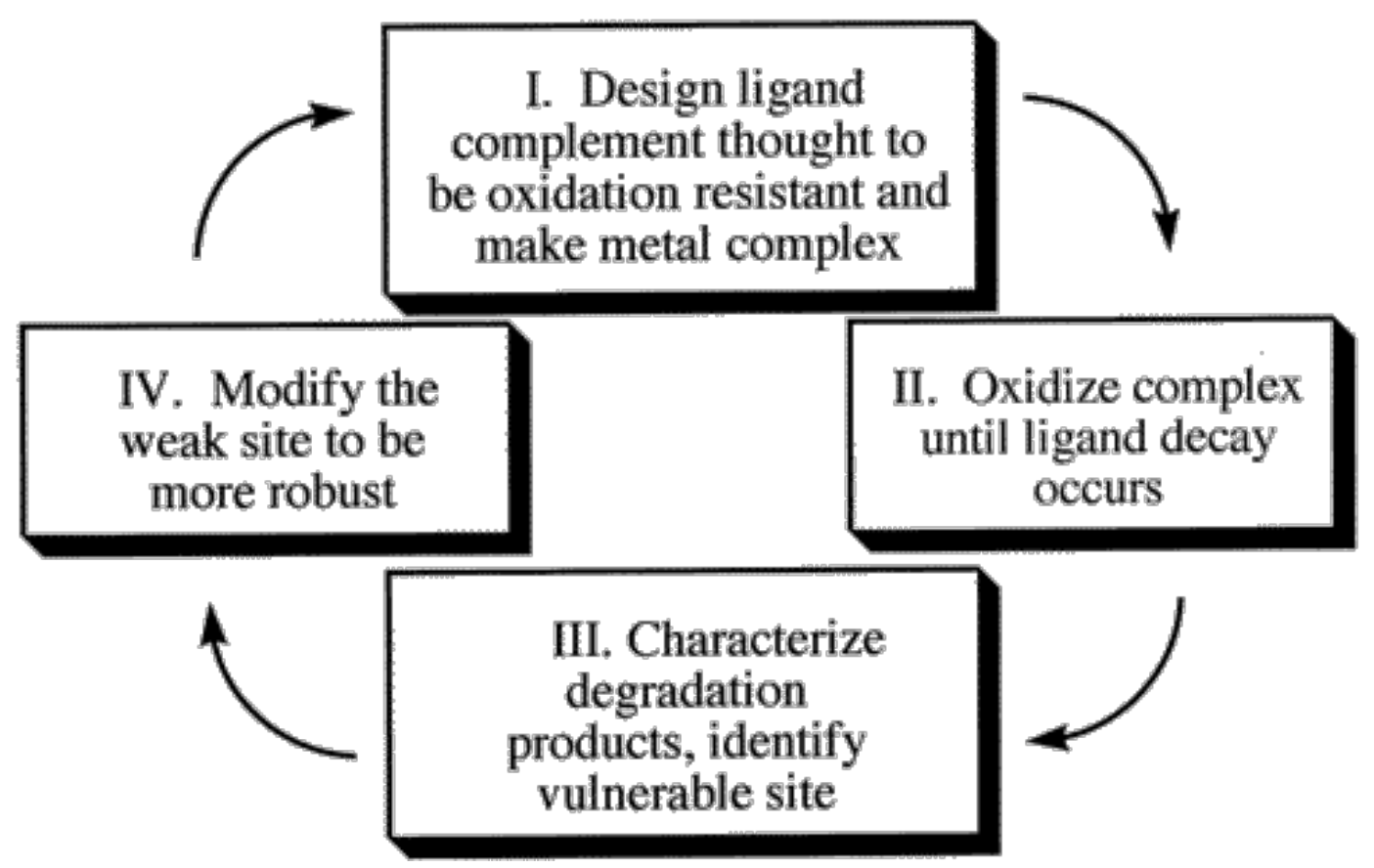

Figure 1.3: Design loop used by Collins to obtain oxidation resistant ligands. Reproduced with permission; from Collins [2] copyright (c) 2002 American Chemical Society. 


\subsection{Applications of TAML Activators in Green Chem- istry}

TAML activators have been reputed as 'dial-a-lifetime-catalyst' since the catalyst lifetime can be controlled. [3, 15] However, the extent at which the TAML catalysts have been selectively designed goes beyond decomposition timescales. This is clearly observed from the variety of applications of the compound. Originally designed to clean up polluted water sources, the usage of TAML has been extended, but not exclusively, to clean up dyes, anthrax and pesticides. $[1,16,17]$

\subsubsection{Pulp and Paper Industry}

Applications in the pulp and paper industry have been explored early in the development of the TAML activators. Collaborations with PAPRO New Zealand and groups at Auckland University, Collins and his team have demonstrated the capacity of iron-TAML complexes to bleach wood pulp by the activation of hydrogen peroxide. [2] Moreover, this could be achieved with small amounts of the TAML material. This application was focused on developing a totally chlorine free technology to remove lignin from pulp and thus, reduce the quantity of organochlorine dumped in the effluent streams. [1] Another use of TAML activators in this area of industry is the treatment of the effluent streams. Work has been done to demonstrate the effective discolouration properties of iron-TAML complexes. [18, 19] One study highlights that TAML/ hydrogen peroxide mix (1.17 ppm TAML activator) can bleach $50-90 \%$ of colour from effluent streams. [3] 


\subsubsection{Textile Industry}

Another leading area of development for TAML activators is the discolouration (Figure 1.4) of effluent stream from dye and textile mills. The research of TAML in this industry is primarily on chlorinated or phenyl containing colourants. [3, 18, 20, 21, 22] The TAML systems used in the textile industry are similar to ones in the pulp and paper industry, showing comparable discolouration of effluent streams.

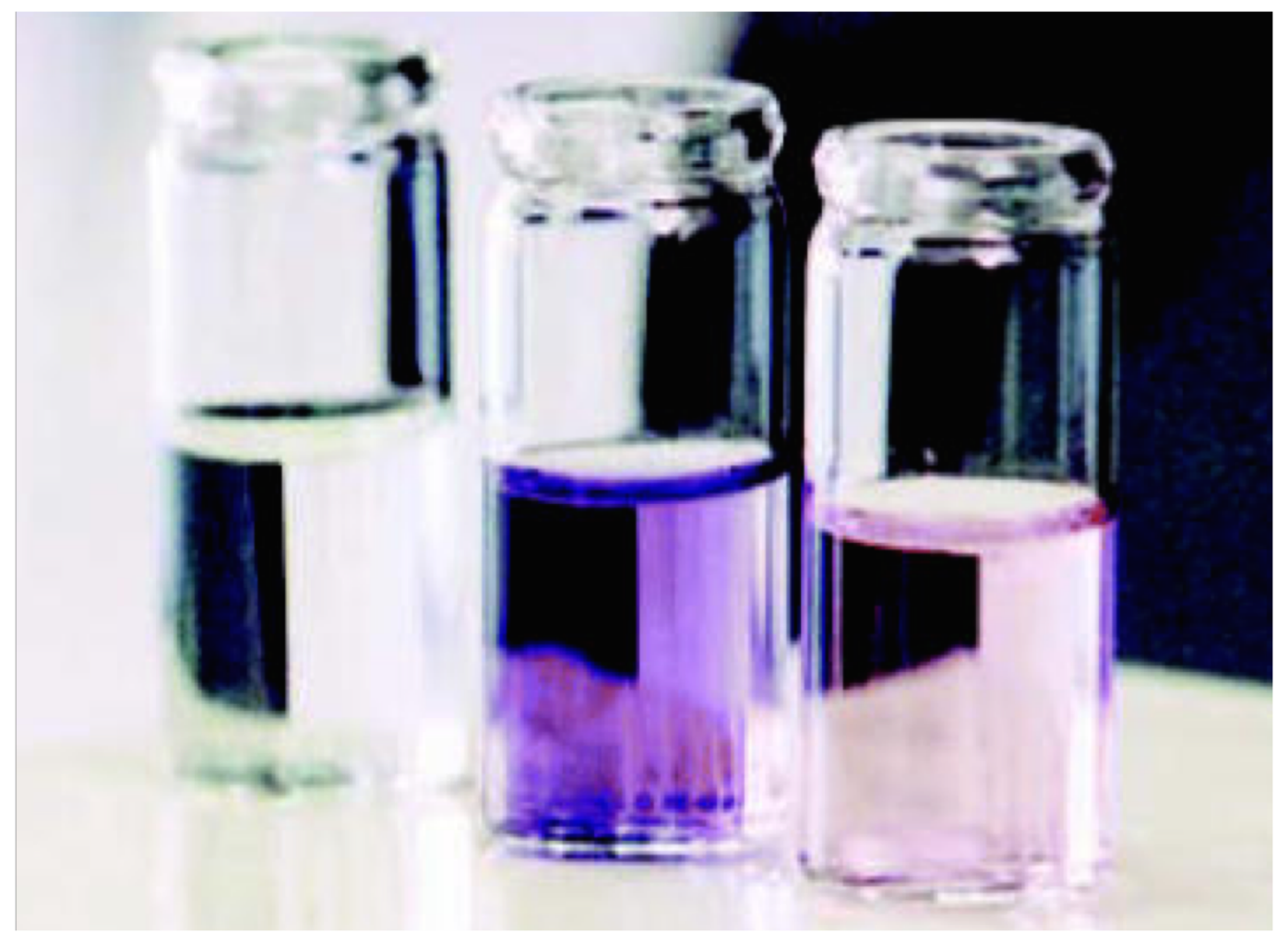

Figure 1.4: Discolouration example. Middle and right vials contain textile dyes, with Fe-TAML added to vial on right. Vial on left contains water for reference. Reproduced from Environmental Health Perspectives [6] 


\subsubsection{Military Applications}

TAML has been tested for military applications in disinfection and detoxification technologies. [1, 19] The selectivity of TAML activators, achieved by changing substituents on the ring or the oxidant used in the reaction, has lead to this widely held view of 'hunter-TAML catalysts' being able to destroy specific pollutants. [2] In the aspect of military use, iron-TAML catalyst have been reported to detoxify trace amounts of trinitrotoluene (TNT) and decontaminate surfaces infected with anthrax spores. [23, 24, 25, 26]

Tests to break down TNT and trinitrobenzene (TNB) have shown that TAML activator/ hydrogen peroxide systems increase the effectiveness of cetyltrimethylammonium bromide surfactants to remove TNT and TNB from contaminated groundwater. The same studies further report that an iron-TAML/ tert-butylhydroperoxide treatment system is even more effective for the breaking down TNT and TNB. [23, 24]

Iron-TAML activators have shown great promise in anthrax decontamination. One study has indicated that TAML can deactivate $\sim 99 \%$ of Bacillus atrophaeus spores (similar to anthrax). [1] The aim for this technology is the implementation of TAML for the decontamination of troops and equipment by simply, spraying the affected the areas with a mixture of iron-TAML, water, baking soda and hydrogen peroxide. [19] Collins and coworkers explain the TAML system which can deactivate the anthrax-like spores to be ironTAML/ peroxide on a surfactant (cetyl trimethylammonium bromide). [26] However, the mechanism of deactivation is yet to be confirmed. [25]

\subsubsection{Desulfurisation of Fuels}

In 2006 the Environmental Protection Agency placed a limit to sulfur content in diesel fuel to less than 15 ppm. [19] Since, dibenzothiophene is the main 
contributing sulfurous compound in diesel fuel, investigations have been performed to determine the reactivity of TAML activators in the aspect of the oxidative breakdown of the dibenzothiophenes from fuel. [27] Collins and coworkers have concluded that the advantage of iron-TAML activators over existing treatments is the small quantities required for desulfurisation to be achieved; the drawback is the complexity of employing the TAML technology, which requires the catalyst to be removed from the fuel due to stability issues. [27]

\subsubsection{Pesticide and Pharmaceuticals}

Pesticides and pharmaceuticals are often used in farming. There are suspected long term effects for continued use; chemicals passing from the environment to other organisms could harm certain natural processes. Such compounds include: fenitrothion (Figure 1.5), other organophosphorus pesticides and estrogen-like compounds. Studies have been performed for the decay of various pesticides and drug compounds by iron-TAML . [6, 28, 29, 30]

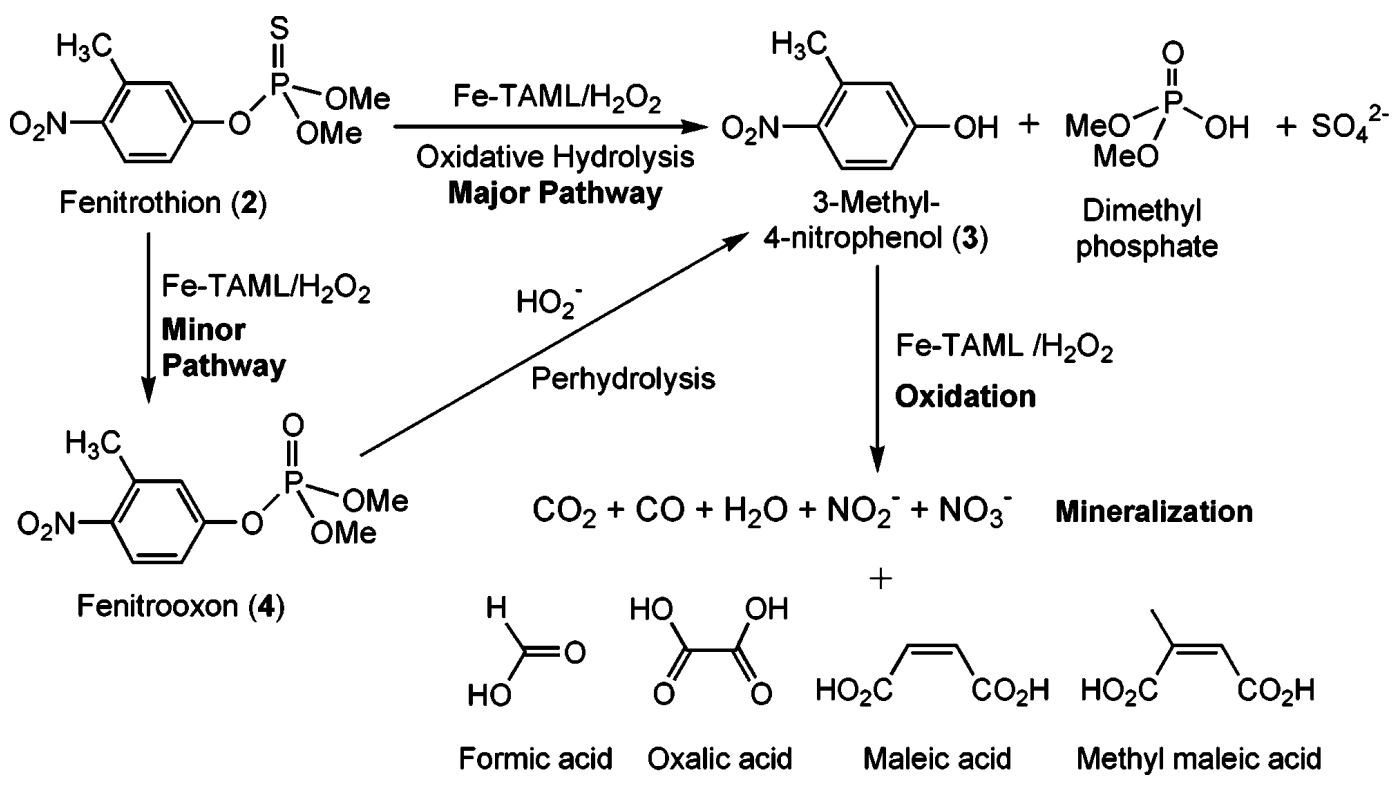

Figure 1.5: Example of pollutant degradation for fenitrothion. Reproduced with permission; from Collins [28] copyright (c) 2006 American Chemical Society. 


\subsubsection{Fast Water Oxidation}

A more recent avenue of application is that of renewable energy production. Ellis et al. has demonstrated that fast water oxidation is achievable with ironTAML complexes. [31] This has implications for developing photochemical technologies for hydrogen production from water.

$$
2 H_{2} O_{(l)} \rightleftharpoons O_{2(g)}+4 H_{(a q)}^{+}+4 e^{-}
$$

However, the reaction (Equation 1.1) is somewhat challenging due to the four proton and four electron transfer that would ultimately lead to dioxygen formation. [24] The conclusions from the study done by Ellis affirm the standing that iron-TAML complexes are an environmentally safe alternative to most water oxidation catalysts, though improvements are required to further the usefulness of the TAML activators. [31]

\subsection{Mechanistic Studies}

Cramer and coworkers then performed a computational study on the mechanism of fast water oxidation using DFT methods and multireference calculations. [32] The study of the fast water oxidation mechanism by Cramer and coworkers builds upon existing knowledge of accessible higher oxidation states for iron oxo complexes. [33, 34, 35, 36, 37] They propose relatively stable intermediate species with electrophilic Fe-O cores which can undergo nucleophilic attack by water.

Cramer's work further proposed a proton coupled electron transfer (PCET) pathway. [32] Though loosely defined by an outer-sphere mechanism, ignoring proximity of charges, the PCET pathway is more energetically feasible than the one electron oxidation or deprotonation pathways. Furthermore, 


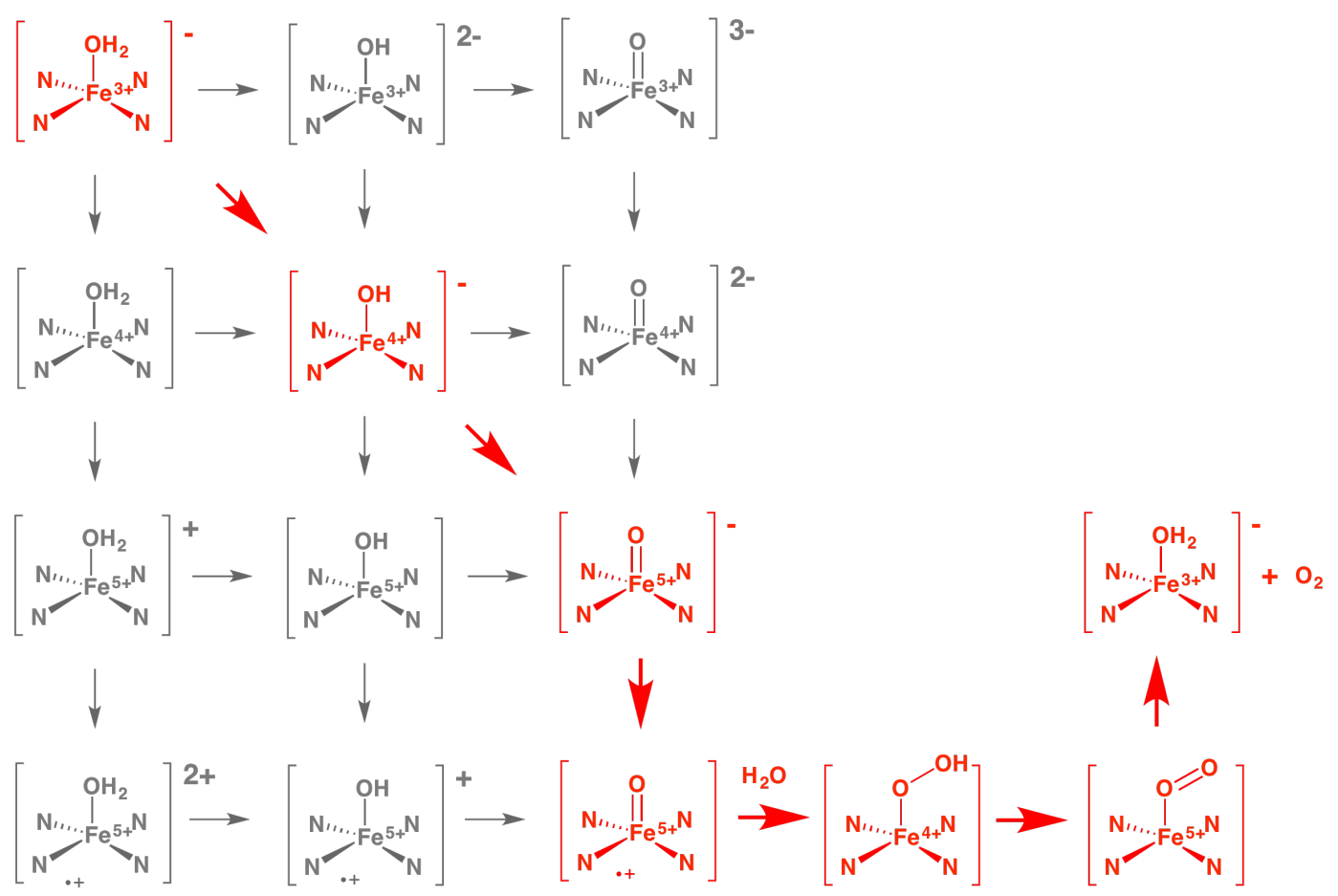

Figure 1.6: Proposed fast water oxidation pathway. Red structures display the proton coupled electron transfer (PCET) pathway along with the water nucleophilic attack and oxygen generation. TAML rings omitted for clarity.

the initially suspected reactive species, $\mathrm{Fe}(\mathrm{V})-\mathrm{O}$, undergoes a one electron oxidation of the TAML ring itself to afford a species which is susceptible to nucleophilic attack by water (Figure 1.6).
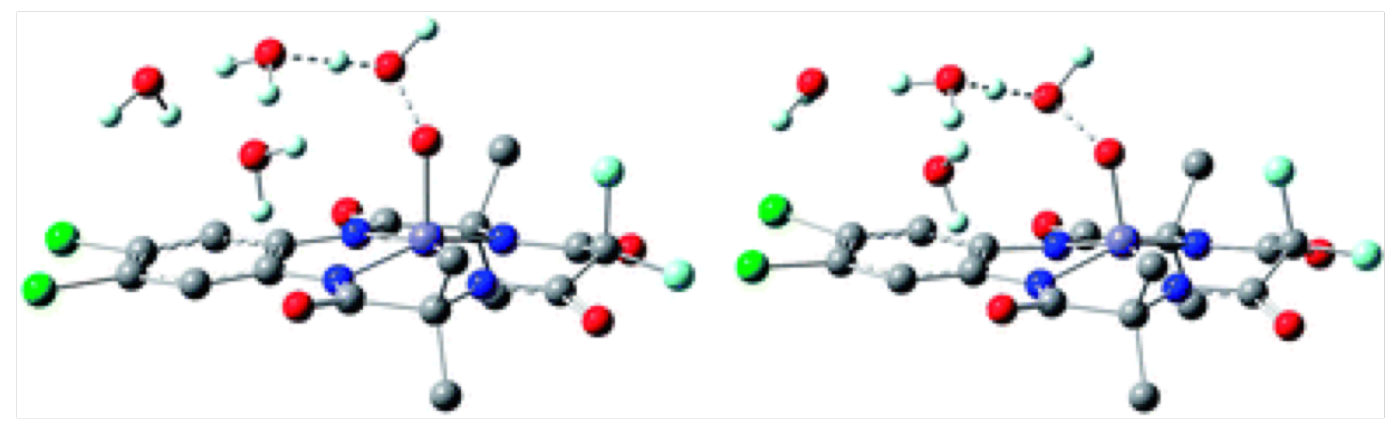

Figure 1.7: Local solvation shell proposed by Cramer. Reproduced from [32] with permission from the PCCP Owner Societies 
The initially suspected reactive species, $\mathrm{Fe}(\mathrm{V})-\mathrm{O}$, was used as a starting point in transition state calculations. It was found that one water molecule is repelled on approach to the electrophilic core. Hence, a local solvation shell (Figure 1.7) was required as to facilitate proton transfer. [32]

With the TAML ligand being oxidised, instead of the metal centre, the reactive intermediate supports a non-innocent TAML ligand which produces some likely instability due to the aromatic radical. In a separate investigation by Ghosh and coworkers, TAML variants with different metal centres have expressed this problem as well. [38]

The focus of this thesis was based around the mechanism of fast water oxidation using Fe(III)-TAML complexes. Furthermore, the work presented will explore how changing various groups on the TAML ring affects the stability of the reactive intermediate. 


\section{Chapter 2}

\section{Methodology}

\subsection{Preamble}

Chemistry deals with the properties, formation and interactions of molecules. Molecules are generally described as being composed of charged particles: positve nuclei and negative electrons. Theoretical chemistry attempts to calculate and subsequently predict structural arrangements, relative energies, electronic properties and interactions of molecules. Since the behaviour of matter at a subatomic scale is impossible to describe using classical physics, quantum mechanics is employed. The equation that is solved to describe the energy in chemical systems using ab-initio methods is the time-independent electronic Schrödinger equation (2.1).

$$
\mathbf{H} \Psi=E \Psi
$$

where, $\mathbf{H}$ is the Hamiltonian operator, $\Psi$ is an electronic wavefunction which describes the electrons of the system and $E$ is the energy of the system. The time-independent electronic Schrödinger equation (2.1) is generally employed for non-dynamic calculations. This uses the Born-Oppenheimer ap- 
proximation, which the nuclei, due to size relative to electrons, are considered "clamped" since quantum nature of nuclei are insignificant. This means that equation 2.1 is solvable by electronic wavefunctions that only depend on the postion and not the momentum of the nuclei. Solving equation 2.1 for a large number of geometries will yield a potential energy surface (PES). From the PES other molecular properties can be calculated by knowing how the energy changes for a given perturbation.

However, the time-independent electronic Schrödinger equation (2.1) can only be solved exactly for chemical systems with one or two particles e.g. the $\mathrm{H}$ atom. The complexity of a many-body system requires Equation 2.1 to be solved by approximation - this is where computational chemistry takes over. Computational chemistry is a subfield of theoretical chemistry in which, mathematical methods are used to compute otherwise complex laws of physics with respect to chemical systems.

There are many methods which may be used to approximate solutions for the time-independent electronic Schrödinger equation (2.1) ranging from low level Hartree-Fock (HF) methods to high levels of accuracy for Full Configuration Interaction (FCI) methods. The selection of method is dependent on the level of accuracy desired as well as the computational cost (i.e. size of the system or time constraints).

\subsubsection{Wavefunction-based Methods}

HF deals with complexity of the many-body system by treating the particles in the system independently. Hence, the energy of system found, in a stepwise fashion where each electron is placed in mean field of all the other electrons, by the average electronic interaction. This is called the self consistent field (SCF) procedure. In HF the SCF procedure and single Slater determinant is used to find the optimal wavefunction (Equation 2.2) to obtain 
the energy of a chemical system. As a result of this mean field description of electronic interaction the HF method cannot encapsulate most chemical phenomena accurately.

$$
\Psi=a_{o} \Phi_{H F}+\sum_{i=1} a_{i} \Phi i
$$

The electronic interaction dealt with in HF is the Pauli principle in which no two electrons can occupy the same molecular orbital and have the same spin - Fermi correlation. Although HF does not fully account for all electronic interactions the HF method can obtain $\sim 99 \%$ of the system energy. However, the remaining $1 \%$ of energy is attributed to the Coulomb correlation (correlation more generally). This is the explicit electron-electron interactions that is only averaged in the HF method but is important to describe the chemistry of the system.

Many methods build upon HF in order to recover this missing correlation. One method employs more excited Slater determinants to the HF wavefunction in a linear scheme which is called Configuration Interaction (CI). Equation 2.3 presents the CI scheme for singles, doubles and triply excited determinants. A similar method, Couple Cluster (CC) uses exponents (Equation 2.4) to generate excited determinants; often truncated to ease computation demand.

$$
\begin{gathered}
\Psi_{C I}=a_{o} \Phi_{H F}+\sum_{i=S} a_{S} \Phi_{S}+\sum_{i=D} a_{D} \Phi_{D}+\sum_{i=T} a_{T} \Phi_{T}+\ldots \\
\Psi_{C C}=e^{T} \Phi_{H F}
\end{gathered}
$$

Another common method is Møller-Plesset (MP) Perturbation Theory (Equation 2.5) which uses the Hamiltonian of HF solution as the first order perturbation the adds further perturbation; the most common being MP2. 


$$
\mathbf{H}=\mathbf{H}_{o}+\lambda \mathbf{H}^{\prime}
$$

The underlying problem with the aforementioned improvements to the HF method, particularly CI and CC methods, is that the computational demand increases drastically for larger and more complex systems.

\subsubsection{Density Functional Theory}

The methods employed in this computational study will be Density Functional Theory (DFT) methods instead of Wavefunction and Perturbative methods. DFT is based on the Hohenberg-Kohn (HK) theorems in which the ground state energy of a system is related to the electron density by some functional. This is vast improvement over wavefunction-based methods as the dimensionality of the $\mathrm{N}$-electron system is reduced from a $4 \mathrm{~N}$ dimensional wavefunction ( 3 spatial and 1 spin-coordinate) to only 4 coordinates (3 spatial and 1 spin-coordinate regardless of the number of electrons). However, the true functional which can perform this is not yet known; thus, approximated functionals are used (of the general form Equation 2.6).

$$
E_{D F T}[\rho]=T_{S}[\rho]+E_{n e}[\rho]+J[\rho]+K[\rho]
$$

Equation 2.6 shows how the HK theorems treat the energy of the system similar to wavefunction-based methods; where $T_{S}[\rho]$ is kinetic energy, $E_{n e}[\rho]$ is the nucleus-electron attraction, and the electron-electron repulsion term can be expanded to include $J[\rho]$ the Coulomb term and $K[\rho]$ the Exchange term. The HK theorems give rise to orbital free DFT which is only a fair description for periodic metallic systems but not molecules. 
To improve DFT for the description of molecules, Kohn and Sham (KS) had to reintroduce orbitals. The once simple 4 coordinate problem had been reverted to a $4 \mathrm{~N}$ problem (similar to HF). Hence, KS DFT is computationally similar to HF but DFT by definition describes the total system from the electron density; only one slater determinant is required.

$$
\begin{aligned}
& E_{D F T}[\rho]=T_{S}[\rho]+E_{n e}[\rho]+J[\rho]+E_{e x}[\rho] \\
& E_{e x}[\rho]=\left(T[\rho]-T_{S}[\rho]\right)+\left(E_{e e}[\rho]-J[\rho]\right)
\end{aligned}
$$

Generally, KS DFT is of the form in Equation 2.7 where the kinetic energy is found from the single Slater-determinant. The exchange-correlation functional (Equation 2.8) is what usually differs between various DFT functionals. This is the underlying problem with DFT. Since the exchange-correlation functional is not known, various parameterisations and approximations are used which are fitted to suit a particular set of systems and not all chemical problems.

In terms of the selection of DFT methods, the concept of the Jacob's ladder of functionals can be considered. [39] One would expect improvements in accuracy for each step of the ladder; Local Density Approximation on the lowest rung, Generalised Gradient Approximation (accounts for the gradient of electron density) on the next, and above that are the Hybrid functionals (mixes HF exchange into exchange-correlation functional). Hence, to achieve accurate results a hybrid functional was considered for the study. 


\subsection{Transition State Theory}

On the PES two minima that correspond to the reactants and products of a chemical reaction are connected by a transition state (TS). Classically, we envisage the TS as the maxima (Figure 2.1) of the intrinsic reaction coordinate (IRC); the one dimension that facilitate the change during the reaction.

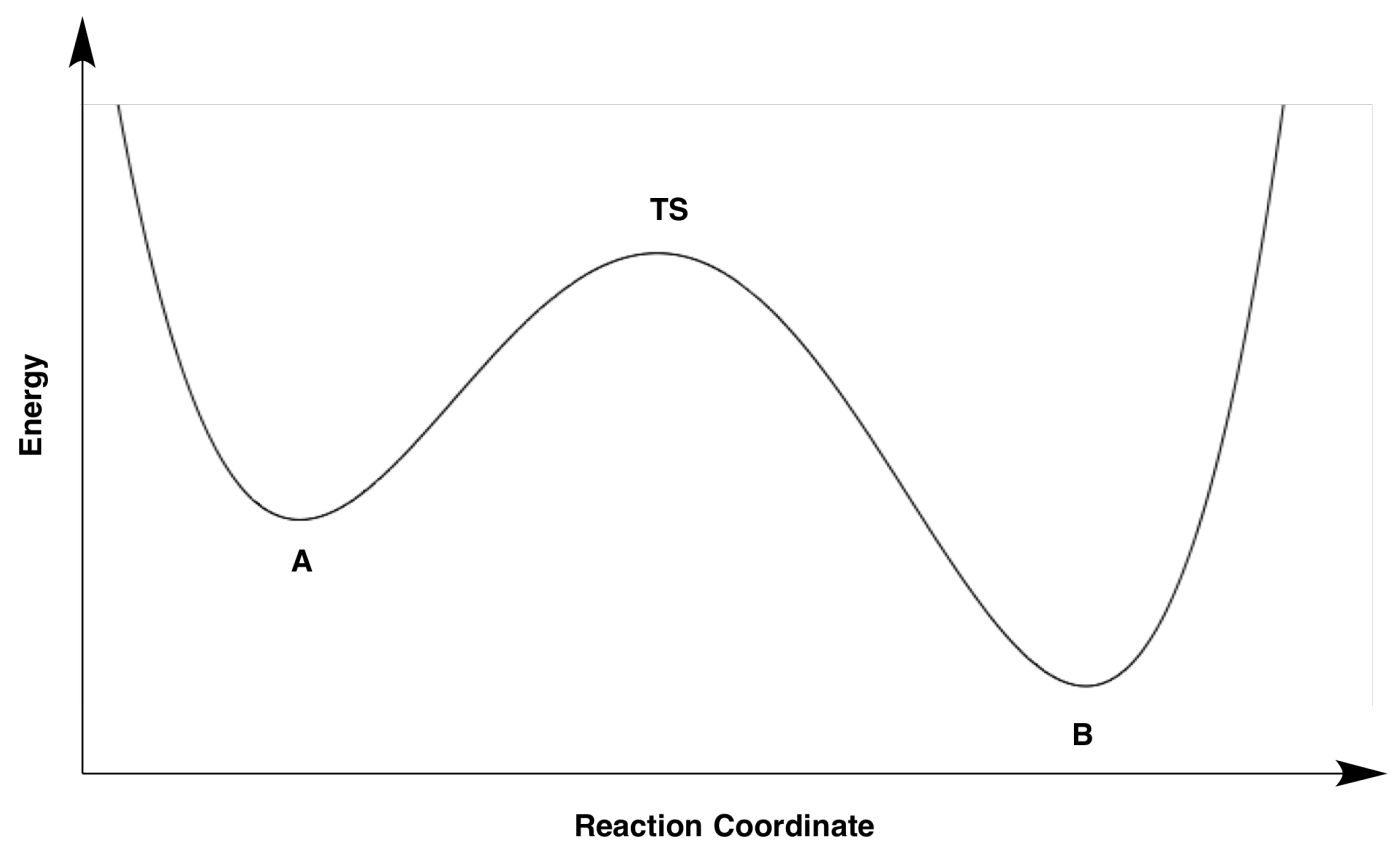

Figure 2.1: Classical representation of a chemical reaction. Reaction proceeds along intrinsic reaction coordinate (IRC).

In this light, finding the transition state of a reaction should then be simple. However, this is only true in concept when one knows the exact coordinate in which the reaction occurs. When all dimensions of the chemical system are considered, the TS is no longer the maxima between two minima but rather a saddle point (Figure 2.2) between two minima. The saddle point is the highest point along the lowest possible energy pathway between two minimum structures and from this the activation energy can be calculated. 


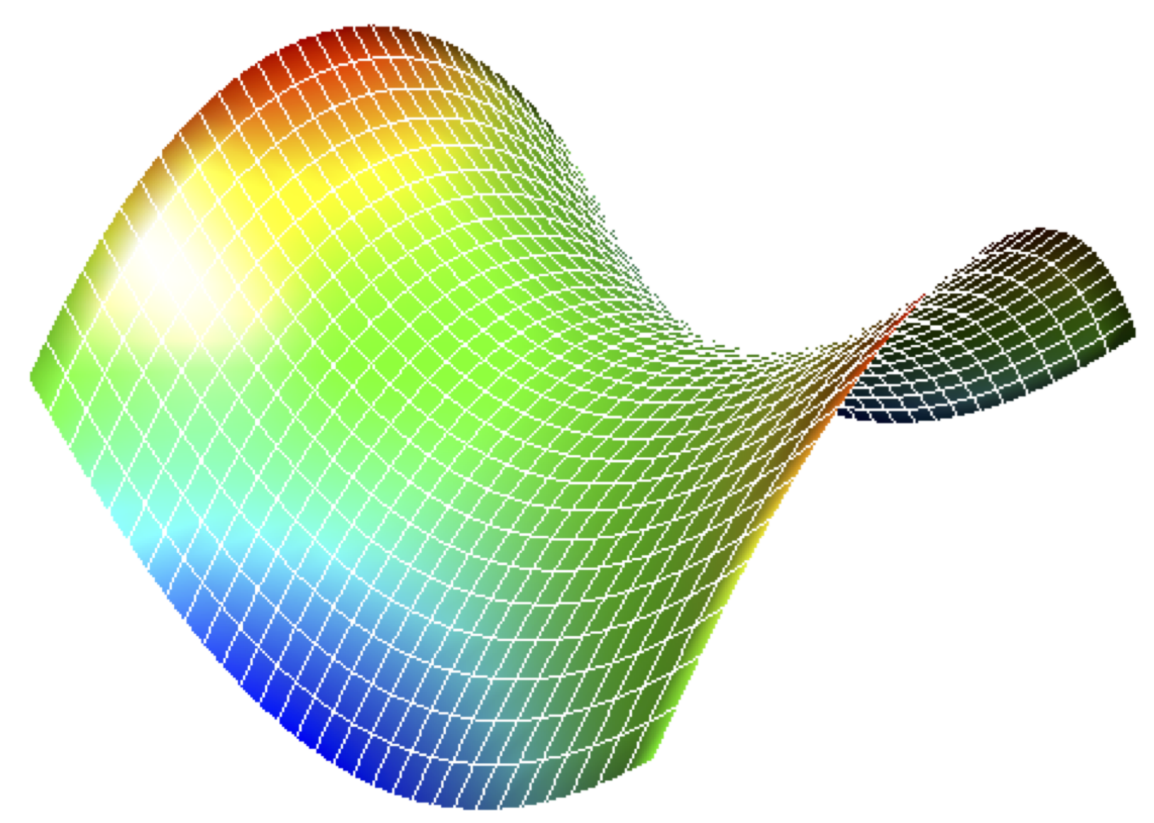

Figure 2.2: Saddle point for illustrative purposes.

The problem now becomes more apparent, in that, the TS is still the maxima along the IRC but a minima along all other coordinates. Hence this stationary point is more difficult to find computationally. Various mathematical methods can be employed to do this; generally, the nature of a suspected TS is confirmed if an imaginary frequency is found (frequencies are computed as the second derivative of the energy).

Computational finesse and chemical intuiton are both required efficiently locate the TS. 


\subsection{Computational Methods}

All DFT calculations were performed using the Gaussian 09 software package. [40] Calculations were run using the hybrid functional PBE0 which includes a $25 \%$ exchange and $75 \%$ correlation weighting. [39, 41, 42] A split valence triple zeta basis set with polarisation functions, Def2-TZVP, was used. $[43,44]$ The nature of the stationary points by geometry optimisations have been determined to be minima by ensuring frequency calculations yielded no imaginary frequencies. Further calculations of particular structures were by way of Natural Bond Orbital (NBO) analysis at the same level of theory and was performed using Gaussian NBO Version 3.1 software package. [45]

Moreover, transition state searches for the water nucleophilic attack mechanism have been run with a local solvation shell of four water molecules, as advised by Cramer's previous work, at the same level of theory as the optimisations. [32] 


\section{Chapter 3}

\section{Results}

Section 1.3.6 introduced the application for iron-TAML activators in the avenue of fast water oxidation. The overall reaction requires the transfer of four protons and four electrons (Equation 1.1). The work presented is based upon the fast water oxidation mechanism proposed in previous mechanistic research. [38, 31, 32, 46]

The main focus of the work is the quantitative modelling of the nucleophilic attack step of the iron-TAML catalysed fast water oxidation mechanism. Furthermore, various substitution patterns on the TAML ring were investigated computationally to understand how to the stabilise of the reactive intermediate and lower the activation barrier of the TS. Hence the mechanism was studied using DFT methods at PBE0/Def2-TZVP level without solvent effects to determine the effects of the structural changes to the energy of the system.

The extremes of inductively electron-withdrawing groups (EWGs) and electrondonating groups (EDGs) were used in the substitution patterns (Figure 3.1) and compared against an experimental structure. 
<smiles></smiles>

A<smiles>O=C1N2N3C(=O)C(F)(C(F)(F)F)N(C(=O)C1(F)F)C(C(F)(F)F)(C(F)(F)F)C(=O)N2c1cc(Cl)c(Cl)cc13</smiles>

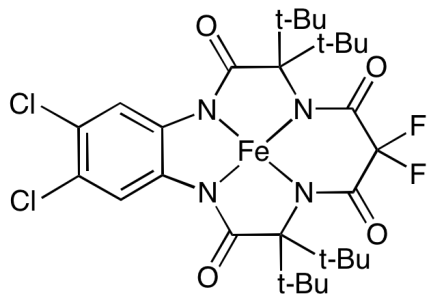

D

B

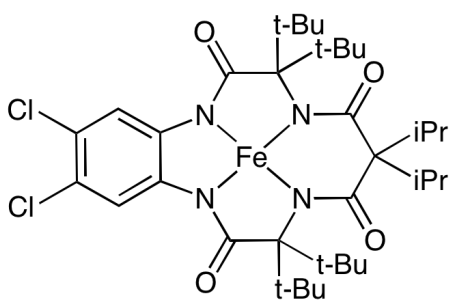

E

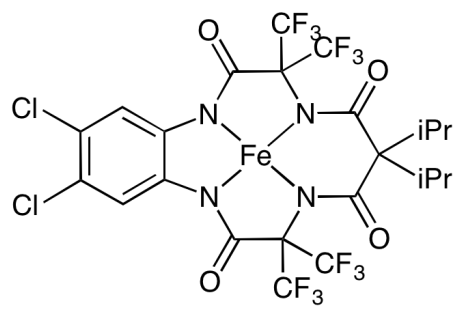

\section{C}

Figure 3.1: TAML structure substitution arrangements. A is an experimental structure. $\mathbf{B}$ is a proposed structure with full electron-withdrawing group (EWG) substitution. $\mathrm{C}$ is a proposed structure with mixture of EWG's and electron-donating groups (EDG). D is a variant structure with mixture of EWG's and electron-donating groups (EDG). $\mathbf{E}$ is the final proposed structure with full electron-donating group (EDG) substitution arrangement.

\subsection{Mechanism Overview}

From Figure 1.6 a reaction scheme was devised to separate the PCET steps in order to fully resolve the mechanism. Figure 3.2 presents the individual steps in the fast water oxidation mechanism catalysed by a general ironTAML activator. Proceeding sections will highlight the various parts of the mechanism in study; PCET steps, TS, and subsequent a PCET for oxygen generation. 

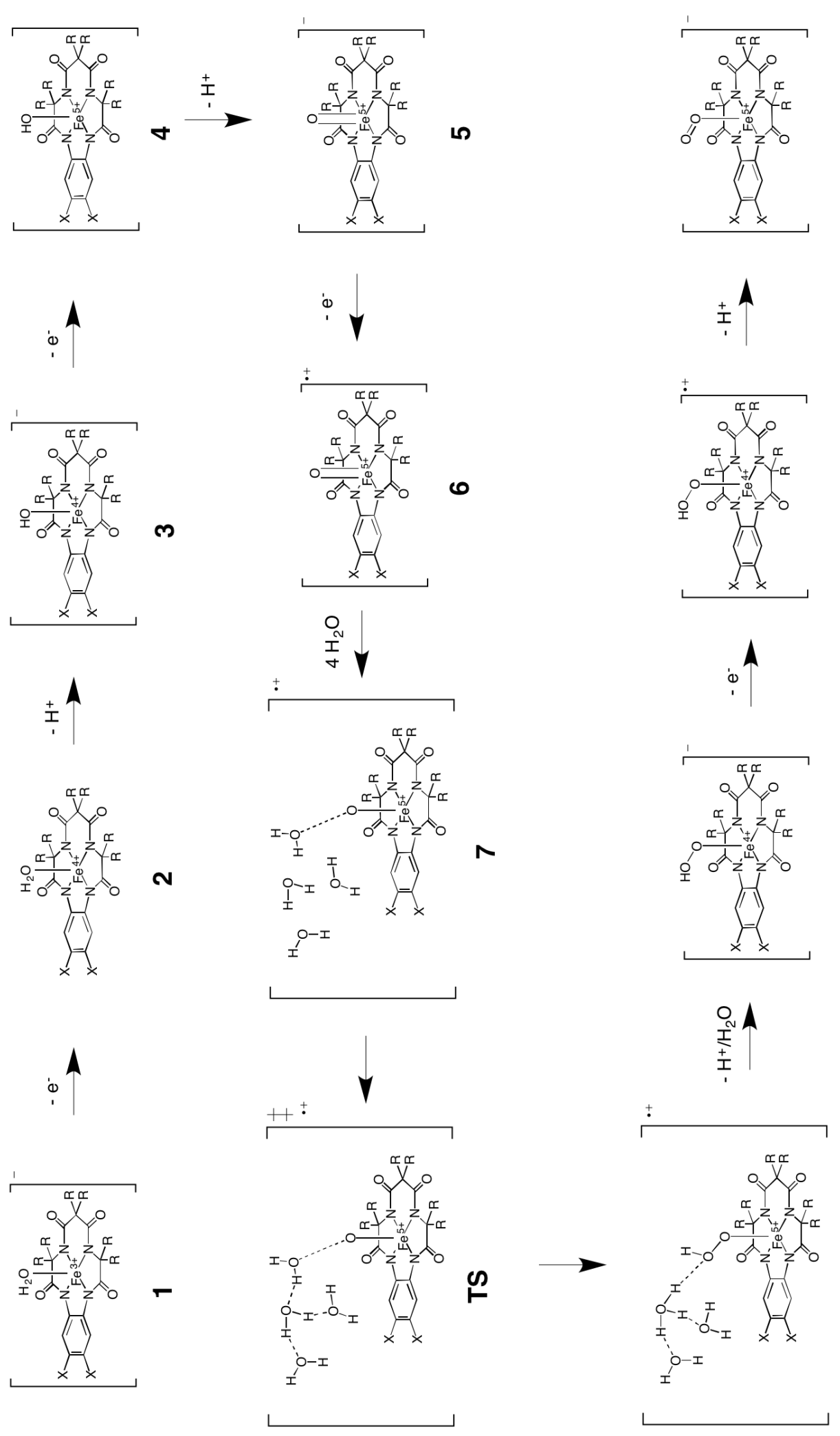

? 


\subsection{Proton-Coupled Electron Transfer Steps}

The proton-coupled electron transfer (PCET) steps were separated into electron loss and proton loss steps. To take into account the free energy of an electron, the redox couple $\mathrm{Li} / \mathrm{Li}^{+}$was calculated to be $-128.42 \mathrm{kcal} \mathrm{mol}^{-1}$ at PBE0/Def2-TZVP. The proton correction calculated at the same level of theory was predicted at $-165.82 \mathrm{kcal} \mathrm{mol}^{-1}$.

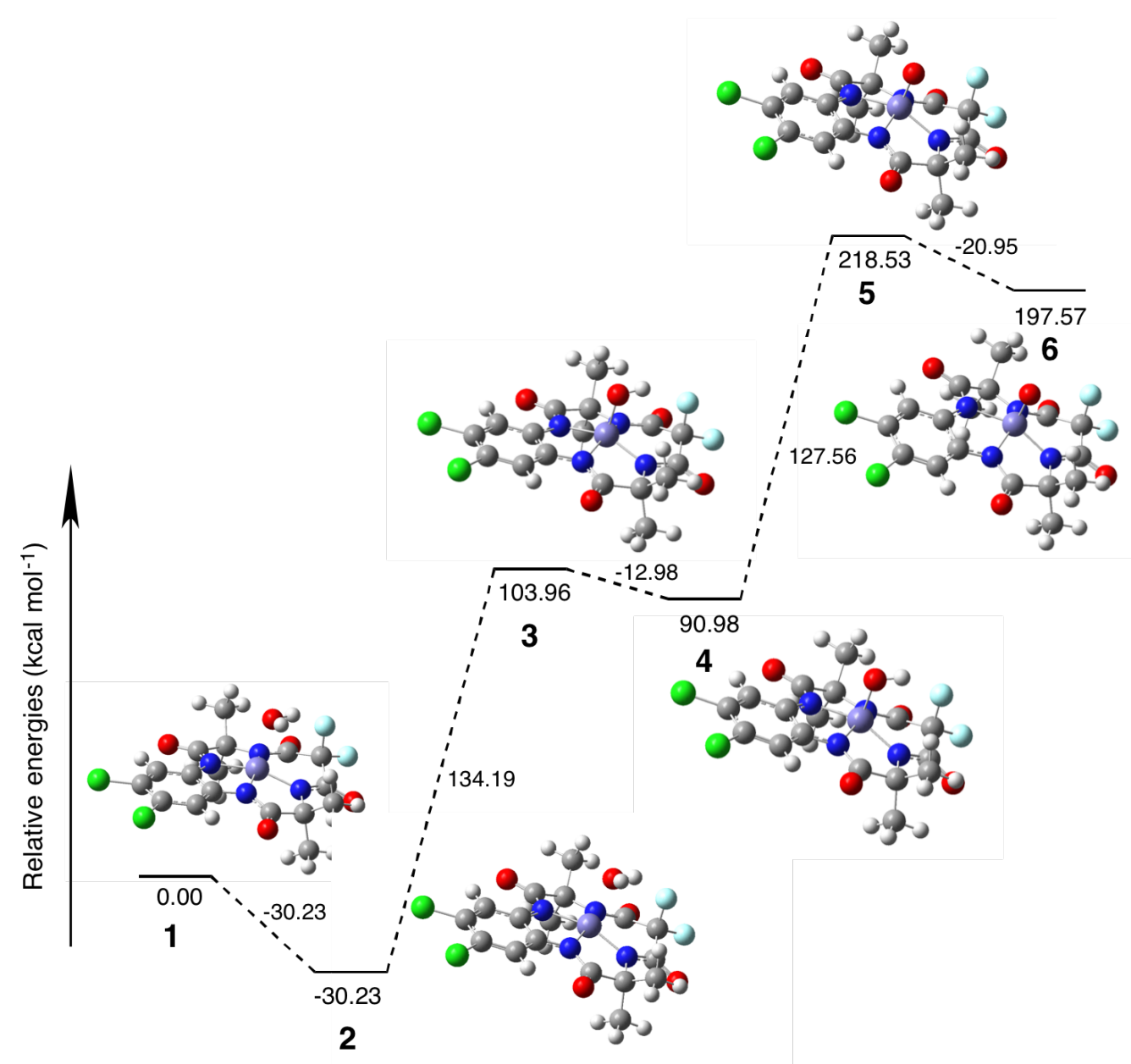

Figure 3.3: PCET steps of mechanism for experimental structure A. Relative energies with respect to starting materials given below structure while energetic differences between steps in the mechanism are given on dotted lines between structure. Calculated at PBE0/Def2-TZVP level of theory. All energies in $\mathrm{kcal} \mathrm{mol}^{-1}$. 
The starting structure (1A) is a negatively charged quartet species. [32] The oxidation of this species to afford 2A (neutral triplet species) is calculated to be exothermic by $30.23 \mathrm{kcal} \mathrm{mol}^{-1}$. (Figure 3.3). Subsequent proton loss marks the first PCET step. The proton loss leads to structure 3A which is higher in energy than the starting materials. The higher energy species is suspected to be due to the omission of solvent correction, thus, charged species will not be stabilised. The work was investigating the effects of particular substitution patterns on the stability of the reactive intermediate and the activation energy of the nucleophilic attack to which solvent effects would not be of benefit. Moreover, once a trend is observed for such substitution patterns the solvent can be optimised for a particular TAML system.

Following Figure 3.3 another PCET step takes place with values -12.98 and $127.56 \mathrm{kcal} \mathrm{mol}^{-1}$ for the oxidation and proton loss respectively. Structure 6A (neutral triplet species) is proposed as the reactive intermediate which is the Fe(IV)-oxo complex where the TAML ring has been oxidised (noninnocent ligand). [38]

The PCET steps were calculated for proposed substitution patterns for the TAML systems (structures B, C, D and E) and are presented in Figure 3.4. It is observed that these PCET steps follow the same trend as the experimental structure A. Furthermore, structures $6 \mathrm{D}$ and $6 \mathrm{E}$ have lower energies than B and $6 \mathrm{C}$ but there is no discernible trend between the substitution patterns and their respective relative energy differences for the other PCET steps in the mechanism. 


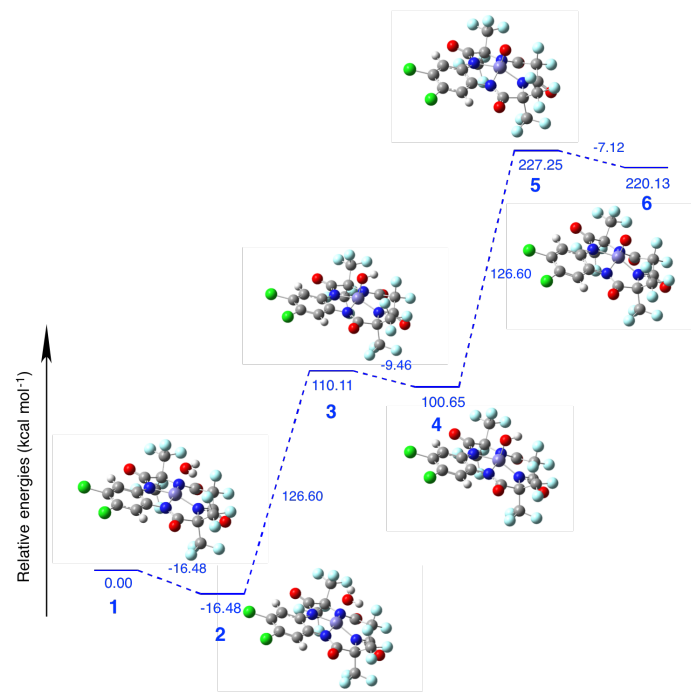

(a) Proposed structure $\mathbf{B}$

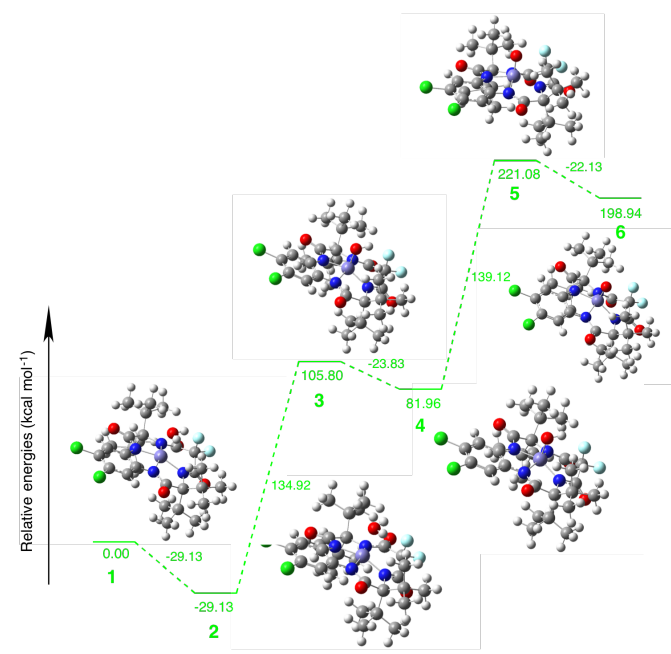

(c) Proposed structure $\mathbf{D}$

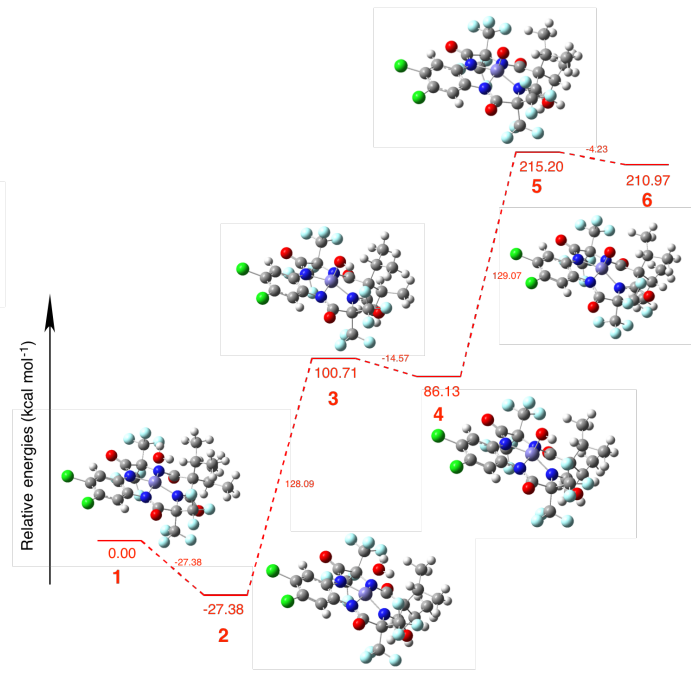

(b) Proposed structure C

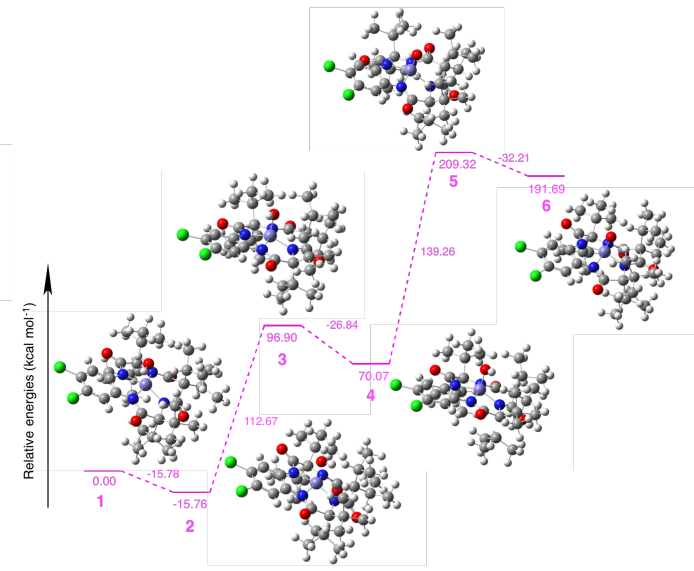

(d) Proposed structure $\mathrm{E}$

Figure 3.4: PCET steps of mechanism for proposed structures D and E. Relative energies with respect to starting materials given below structure while energetic differences between steps in the mechanism are given on dotted lines between structure. Calculated at PBE0/Def2-TZVP level of theory. All energies in $\mathrm{kcal} \mathrm{mol}^{-1}$. For a more comprehensive list of relative energies refer to Tables 3.1 and 3.2 


\subsection{Transition State: Water Nucleophilic Attack}

The TS of the iron-TAML catalysed fast water oxidation was calculated via a local solvation shell. Four water molecules were placed around the reactive intermediate in fashion as aforementioned in Section 1.4. One of the water molecule is the suspected nucleophile and the remaining three water molecules act as a proton relay which solvates the hydronium ion produce in the reaction. Figure 3.5 illustrates a successful nucleophilic attack of a water molecule on the electrophilic Fe-oxo core of the experimental TAML structure (A). The free energy of activation was determined to be $16.61 \mathrm{kcal} \mathrm{mol}^{-1}$ at PBE0/Def2-TZVP level with reaction proceeding in exothermic fashion.

Likewise, the TS for proposed structure B (full EWG substitution arrangement) was modelled successfully with the activation barrier predicted at 9.12 kcal mol ${ }^{-1}$. Comparison of Figure 3.5 and Figure 3.6 shows a similar reorientation of the local solvation shell from step 7 through to 8 . Moreover, structure D (Figure 3.7) follows the same trend with an lower Gibb's free energy value of $7.94 \mathrm{kcal} \mathrm{mol}^{-1}$. (Figures 3.5, 3.6, 3.7 and 3.8 are enlarged for clarity and the molecules rotated to show local solvation rearrangements.)

It is duly noted that the TS for $\mathbf{C}$ (Figure 3.8 ) and $\mathbf{E}$ corresponds to the substitution arrangement with iso-propyl groups on the end of the TAML systems. Close inspection of Figure 3.8 reveals a repulsion of the solvation shell. Additionally, the TS for $\mathbf{E}$ was attempted, more details in this regard in next chapter. 


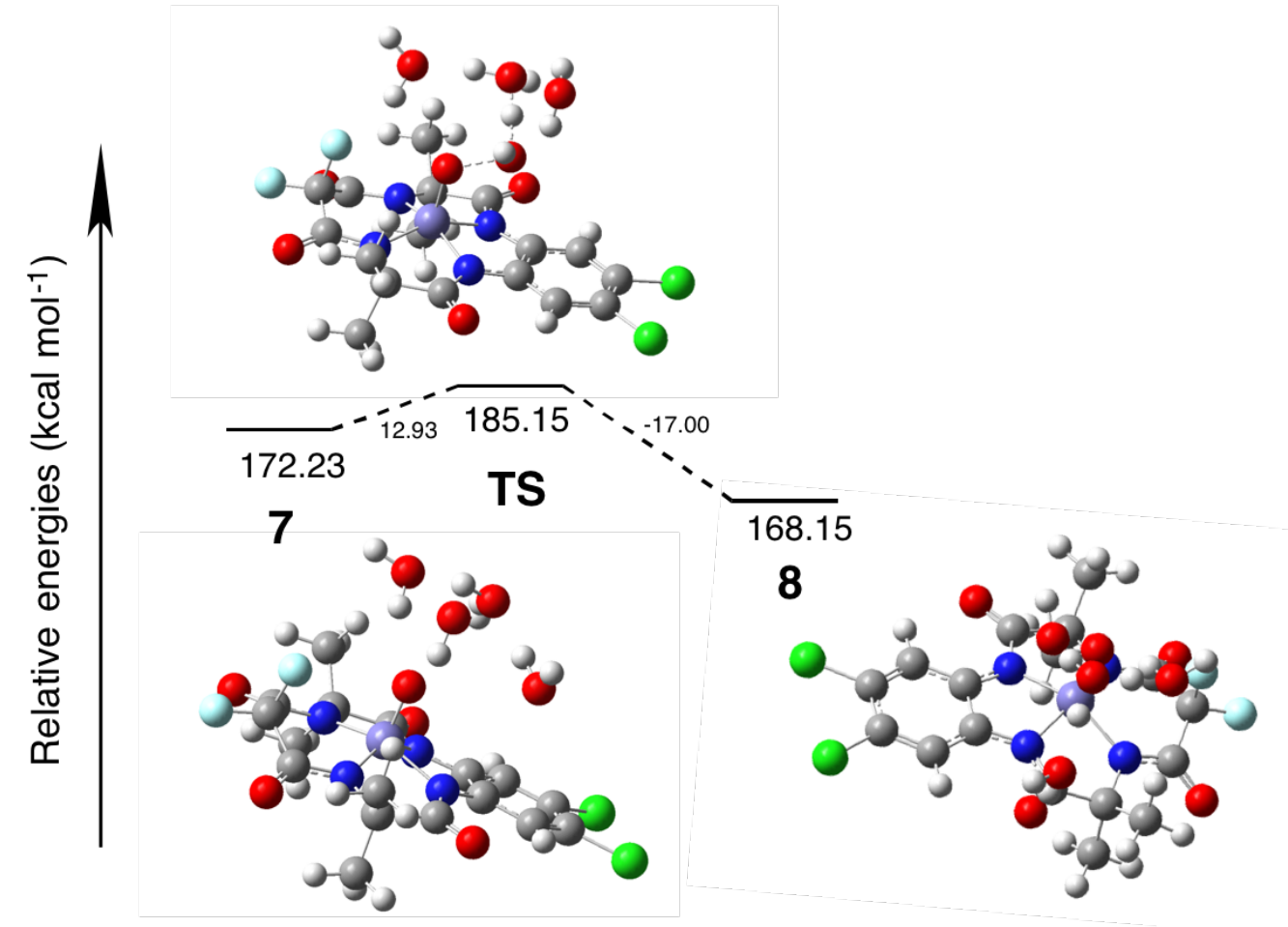

Figure 3.5: Nucleophilic attack of water on the reactive intermediate 6A. Four water molecule local solvation shell present to facilitate proton transfers and solvation of the latter hydronium ion. Relative energies with respect to starting materials given below structure while energetic differences between steps in the mechanism are given on dotted lines between structure. Calculated at PBE0/Def2-TZVP level of theory. All energies in $\mathrm{kcal} \mathrm{mol}^{-1}$. 


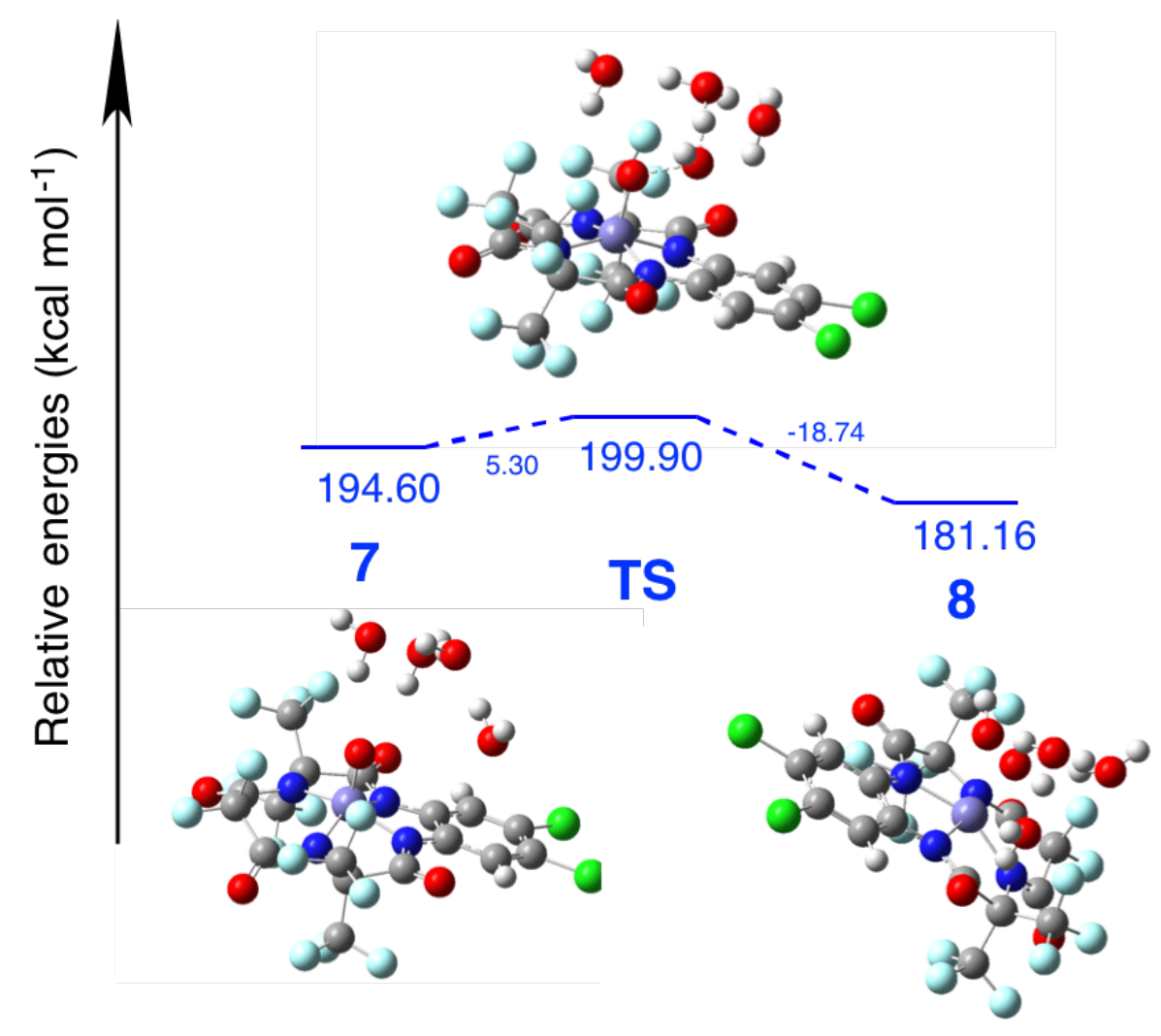

Figure 3.6: Nucleophilic attack of water on the reactive intermediate 6B. Four water molecule local solvation shell present to facilitate proton transfers and solvation of the latter hydronium ion. Relative energies with respect to starting materials given below structure while energetic differences between steps in the mechanism are given on dotted lines between structure. Calculated at PBE0/Def2-TZVP level of theory. All energies in $\mathrm{kcal} \mathrm{mol}^{-1}$. 


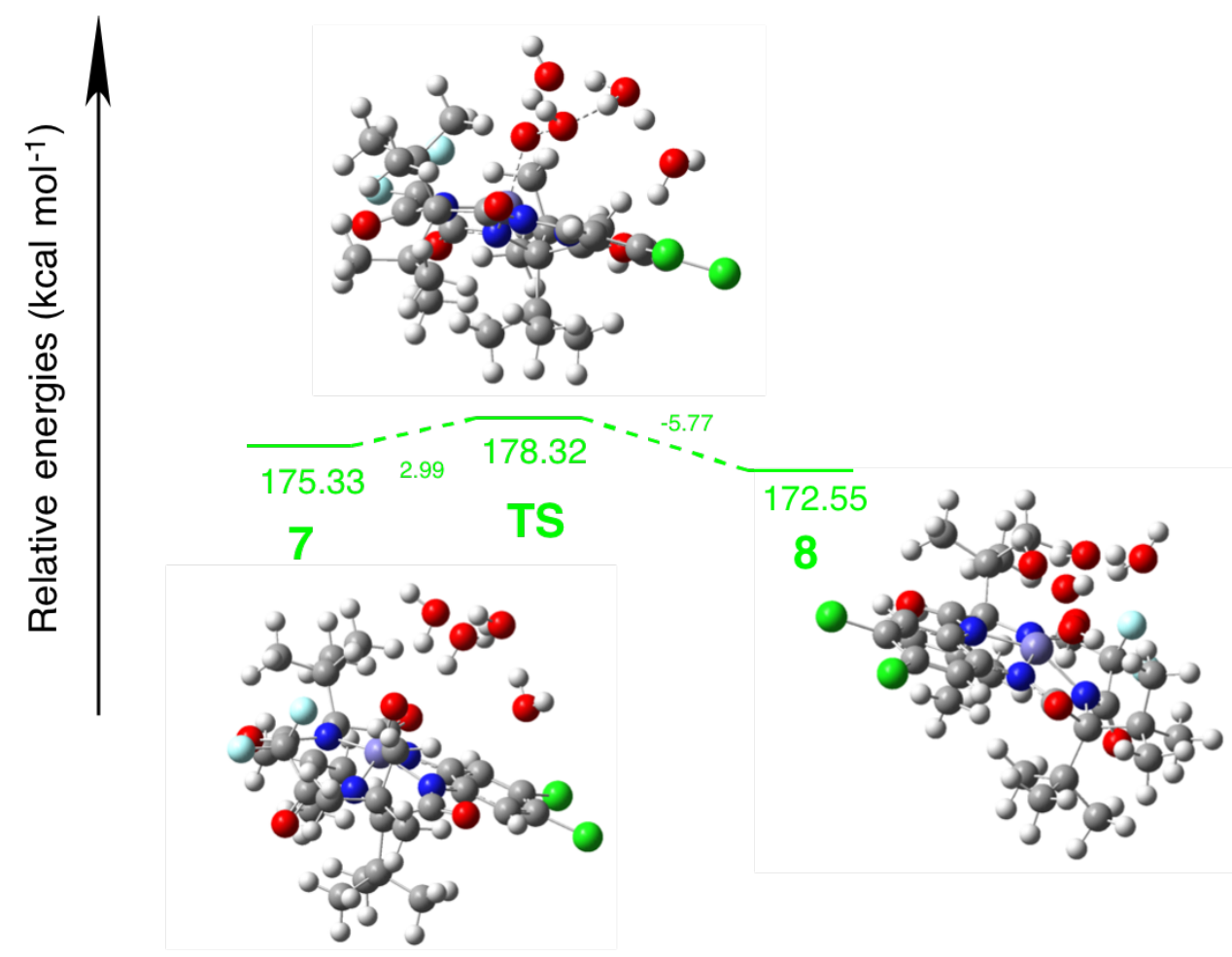

Figure 3.7: Nucleophilic attack of water on the reactive intermediate 6D. Four water molecule local solvation shell present to facilitate proton transfers and solvation of the latter hydronium ion. Relative energies with respect to starting materials given below structure while energetic differences between steps in the mechanism are given on dotted lines between structure. Calculated at PBE0/Def2-TZVP level of theory. All energies in $\mathrm{kcal} \mathrm{mol}^{-1}$. 


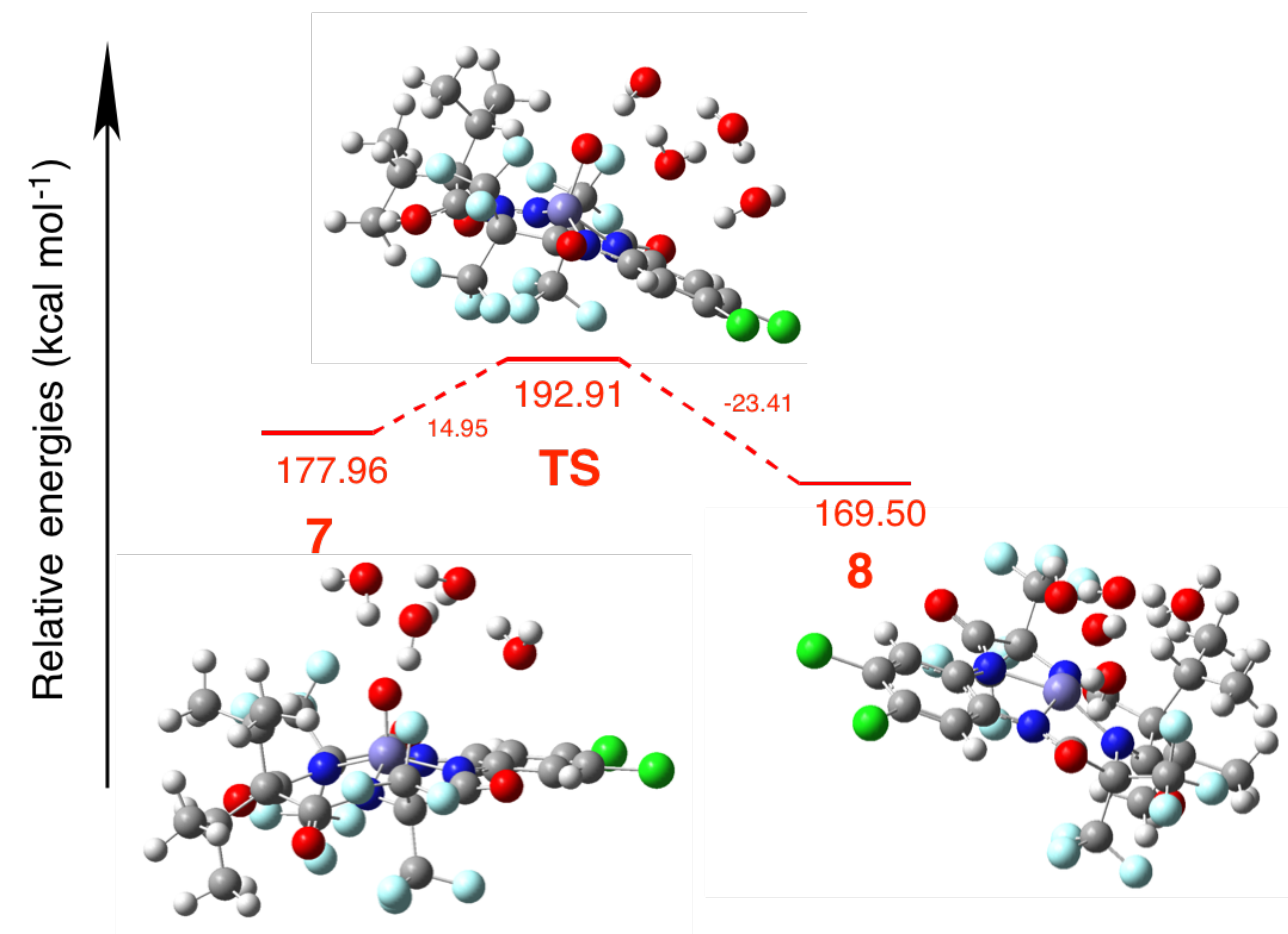

Figure 3.8: Nucleophilic attack of water on the reactive intermediate 6C. Four water molecule local solvation shell present to facilitate proton transfers and solvation of the latter hydronium ion. Relative energies with respect to starting materials given below structure while energetic differences between steps in the mechanism are given on dotted lines between structure. Calculated at PBE0/Def2-TZVP level of theory. All energies in $\mathrm{kcal} \mathrm{mol}^{-1}$.

\subsection{Oxygen Generation}

Figure 3.9 presents the results for the oxygen generation steps of the experimental structure A. After the nucleophilic attack, a ferryl peroxide species is formed 8. Structure 9 was determined as the ferryl peroxide species with the removal of the local solvation shell which at this stage is three water molecules solvating a hydronium ion (product of the nucleophilic attack). Evidently, loss of the solvation shell raises the energy of the system (species is negatively charged) and further justifies the higher energy species is precedent PCET steps (Section 3.2). 


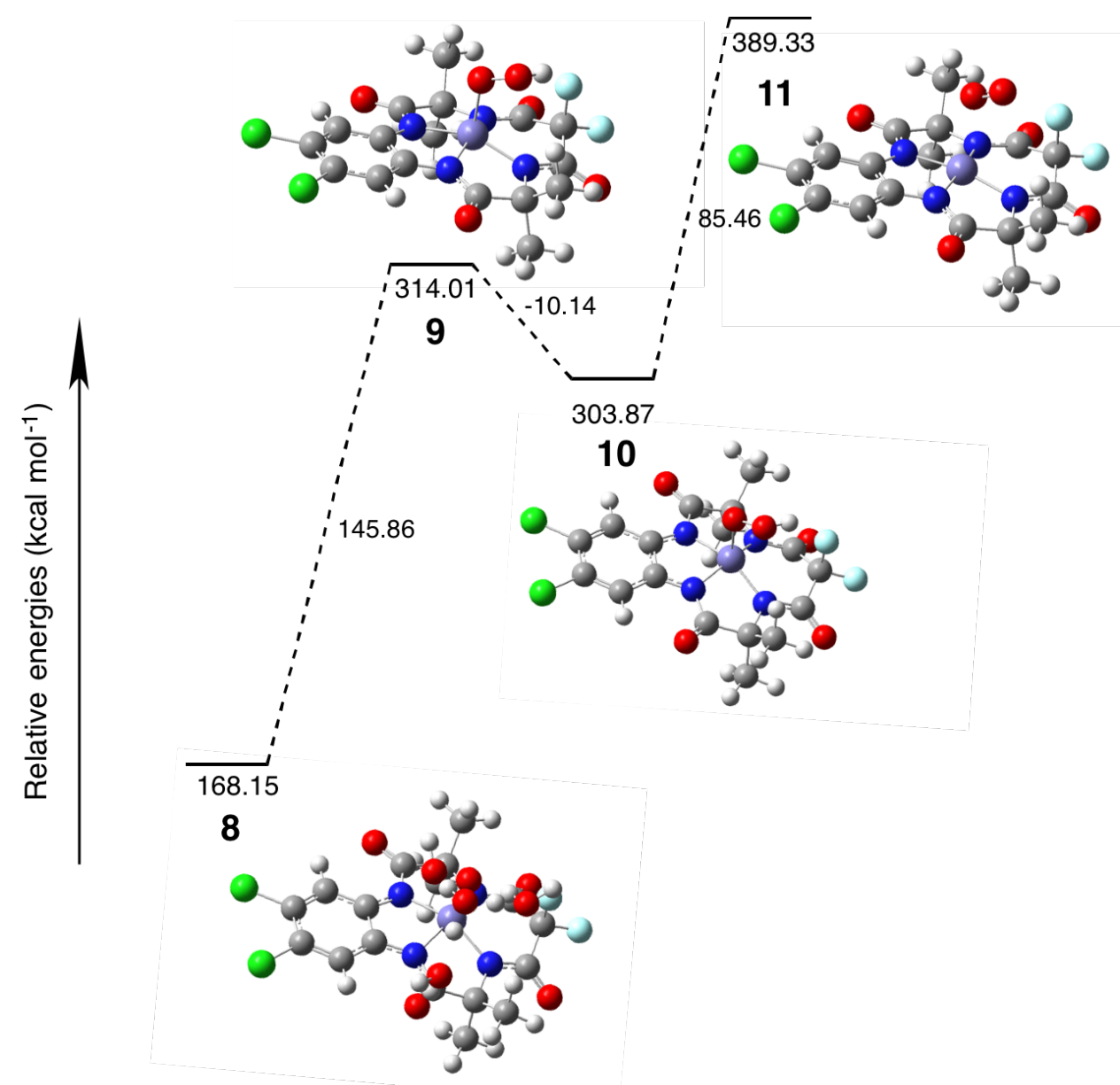

Figure 3.9: Oxygen generation steps of the mechanism for experimental structure A. Relative energies with respect to starting materials given below structure while energetic differences between steps in the mechanism are given on dotted lines between structure. Calculated at PBE0/Def2-TZVP level of theory. All energies in kcal mol ${ }^{-1}$. 
The energetic differences for steps $\mathbf{9}$ to $\mathbf{1 1}$ is similar the loss of a electron and loss of a proton (PCET) which agrees with prior mechanistic studies. [32, 46] These steps were indeed corrected for the electron loss (redox couple $\mathrm{Li} / \mathrm{Li}^{+}$ calculated to be $-128.42 \mathrm{kcal} \mathrm{mol}^{-1}$ at PBE0/Def2-TZVP) and proton loss (at the same level of theory, $-165.82 \mathrm{kcal} \mathrm{mol}^{-1}$ ).

The oxidation step from 9 (negatively charged triplet species) to 10 (neutral doublet species) had an energy difference predicted at $-10.14 \mathrm{kcal} \mathrm{mol}^{-1}$. The loss of the proton from 10 to 11 generates molecular oxygen; this step has a calculated energy difference of $85.46 \mathrm{kcal} \mathrm{mol}^{-1}$.

The PCET steps for oxygen generation were calculated for the proposed substitution patterns for the TAML systems (structures B, C, D and E) and herein are the results (Figure 3.10). As with previous PCET steps, no discernible trend for the substitution effects is apparent from the energy differences. These final steps of the fast water oxidation mechanism are not considered as the rate-determining steps according to prior studies. [32, 46]

Full mechanisms for all the iron-TAML catalysed fast water oxidation reactions studied in this work are delineated in Appendix C. 


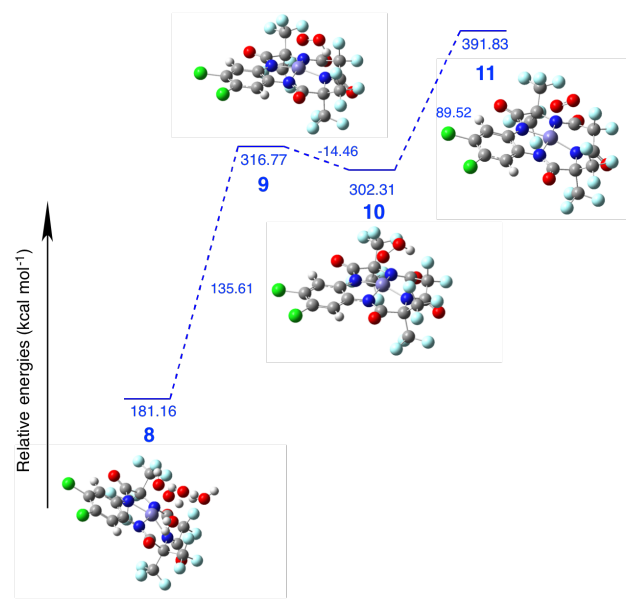

(a) Proposed structure B

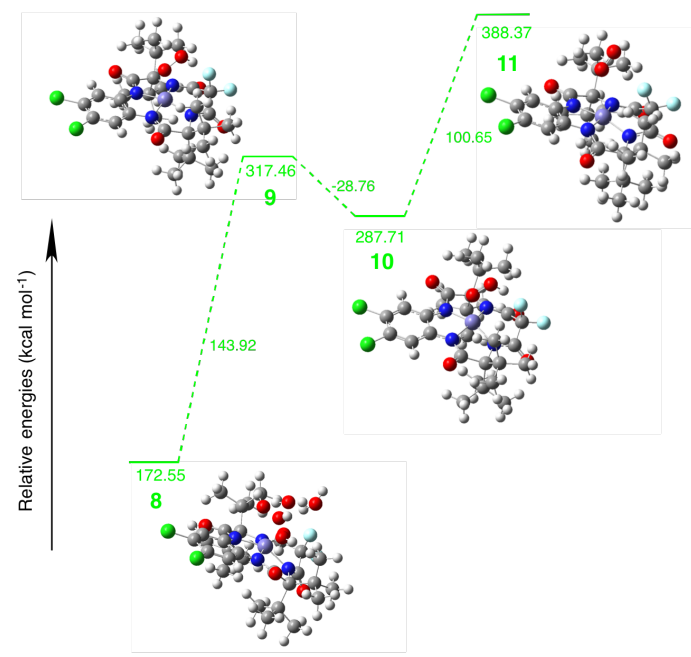

(c) Proposed structure D

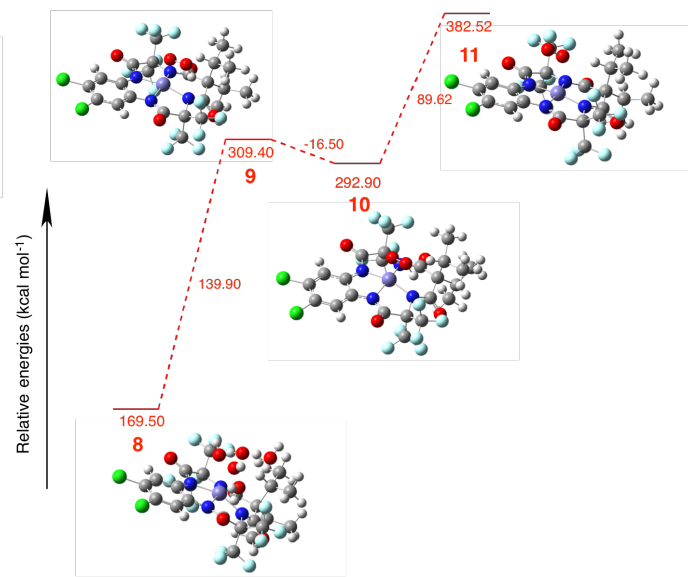

(b) Proposed structure C

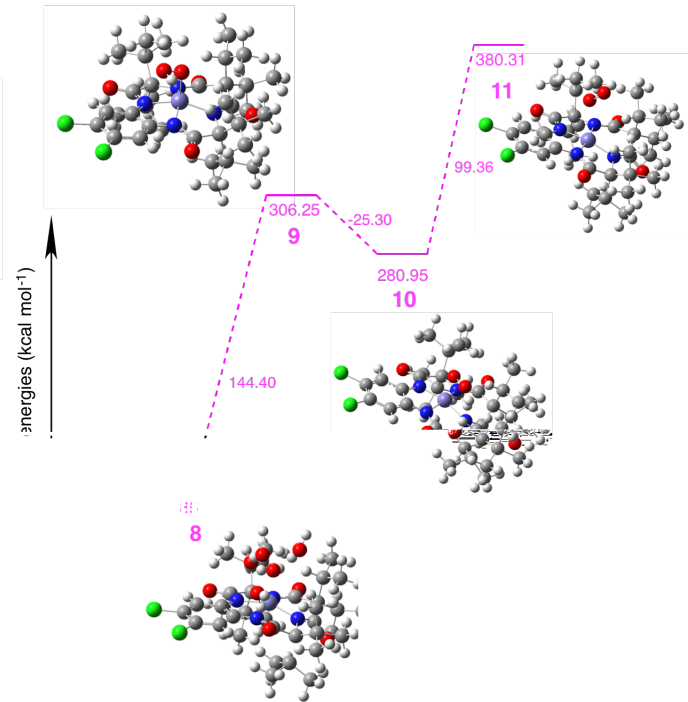

(d) Proposed structure E

Figure 3.10: Oxygen generation steps of the mechanism for proposed structure B. Relative energies with respect to starting materials given below structure while energetic differences between steps in the mechanism are given on dotted lines between structure. Calculated at PBE0/Def2-TZVP level of theory. All energies in $\mathrm{kcal} \mathrm{mol}^{-1}$. For a more comprehensive list of relative energies refer to Tables 3.1 and 3.2 
Table 3.1: Relative energies with respect to previous structure. Calculated at PBE0/Def2-TZVP level of theory. All energies in $\mathrm{kcal} \mathrm{mol}^{-1}$.

\begin{tabular}{lrrrrr}
\hline & \multicolumn{1}{c}{$\mathrm{A}$} & \multicolumn{1}{c}{$\mathrm{B}$} & \multicolumn{1}{c}{$\mathrm{C}$} & \multicolumn{1}{c}{$\mathrm{D}$} & \multicolumn{1}{c}{$\mathrm{E}$} \\
\hline 1 & 0.00 & 0.00 & 0.00 & 0.00 & 0.00 \\
2 & -30.23 & -16.48 & -27.38 & -29.13 & -15.76 \\
3 & 134.19 & 126.59 & 128.09 & 134.92 & 112.67 \\
4 & -12.98 & -9.46 & -14.58 & -23.83 & -26.84 \\
5 & 127.56 & 126.60 & 129.07 & 139.12 & 139.26 \\
6 & -20.95 & -7.12 & -4.23 & -22.13 & -17.63 \\
7 & -25.35 & -25.53 & -33.01 & -23.61 & -32.21 \\
TS & 12.93 & 5.30 & 14.95 & 2.99 & \\
8 & -17.00 & -18.74 & -23.40 & -5.77 & 2.37 \\
9 & 145.86 & 135.61 & 139.90 & 143.92 & 144.40 \\
10 & -10.14 & -14.46 & -16.50 & -28.76 & -25.30 \\
11 & 85.46 & 89.52 & 89.62 & 100.65 & 99.36 \\
\hline
\end{tabular}


Table 3.2: Relative energies with respect to starting material. Calculated at PBE0/Def2-TZVP level of theory. All energies in $\mathrm{kcal} \mathrm{mol}^{-1}$.

\begin{tabular}{lrrrrr}
\hline & \multicolumn{1}{c}{$\mathrm{A}$} & \multicolumn{1}{c}{$\mathrm{B}$} & \multicolumn{1}{c}{$\mathrm{C}$} & $\mathrm{D}$ & \multicolumn{1}{c}{$\mathrm{E}$} \\
\hline 1 & 0.00 & 0.00 & 0.00 & 0.00 & 0.00 \\
2 & -30.23 & -16.48 & -27.38 & -29.13 & -15.76 \\
3 & 103.96 & 110.11 & 100.71 & 105.79 & 96.90 \\
4 & 90.98 & 100.65 & 86.13 & 81.96 & 70.07 \\
5 & 218.53 & 227.25 & 215.20 & 221.08 & 209.32 \\
6 & 197.58 & 220.13 & 210.98 & 198.94 & 191.69 \\
7 & 172.23 & 194.60 & 177.96 & 175.33 & 159.48 \\
$\mathrm{TS}$ & 185.15 & 199.90 & 192.91 & 178.32 & \\
8 & 168.15 & 181.16 & 169.50 & 172.55 & 161.85 \\
9 & 314.01 & 316.77 & 309.41 & 316.48 & 306.23 \\
10 & 303.87 & 302.31 & 292.90 & 287.71 & 280.95 \\
11 & 389.33 & 391.83 & 382.52 & 388.37 & 380.31 \\
\hline
\end{tabular}




\section{Chapter 4}

\section{Discussion}

\subsection{Comparison of Results}

To ensure the result obtained are consistent with precedent computational work a comparison is presented on Table 4.1. The values below from Cramer are calculated at M06L/6-31+G(d) level of theory with ECP10MDF SDD pseudo-potential on Fe atom. [32] These values are for the experimental structure $\mathbf{A}$ and are presented without the solvent correction to allow for comparison with our results at PBE0/Def2-TZVP.

The free energy of an electron was obtained from a $\mathrm{Na} / \mathrm{Na}^{+}$redox couple instead of the $\mathrm{Li} / \mathrm{Li}^{+}$redox couple used in previous chapter due to SCF issues for the Li atom at the M06L/6-31+G(d) level. The free energy of an electron was predicted at M06L/6-31+G(d) level to be $-104.75 \mathrm{kcal} \mathrm{mol}^{-1}$ and $-121.77 \mathrm{kcal} \mathrm{mol}^{-1}$ at PBE0/Def2-TZVP level. Likewise, the free energy of a proton was obtained. This was calculated to be $-162.76 \mathrm{kcal} \mathrm{mol}^{-1}$ at M06L/6-31+G(d) level of theory and $-165.82 \mathrm{kcal} \mathrm{mol}^{-1}$ at PBE0/Def2-TZVP level of theory. These values were used to correct the electron and proton losses for the PCET steps of the mechanism. 
Interestingly, the direction (positive or negative) and magnitude of each step is relatively similar; albeit $\sim 4-60 \mathrm{kcal} \mathrm{mol}^{-1}$ difference. Moreover, the TS values presented may not be an accurate comparison. This is due to the lack of data from Cramer for structures 7A and 8A ( $\Delta \mathrm{G}$ value comparison provided in further sections).

Table 4.1: Relative energies of experimental structure A with respect to starting material. Comparing PBE0/Def2-TZVP results with those of Cramer (M06L/6-31+G(d) with ECP10MDF SDD pseudo-potential on Fe). [32]. All energies in $\mathrm{kcal} \mathrm{mol}^{-1}$.

\begin{tabular}{lrr}
\hline & \multicolumn{1}{c}{ A } & Cramer \\
\hline 1 & 0.00 & 0.00 \\
3 & 110.61 & 122.68 \\
4 & -6.32 & -10.31 \\
5 & 127.56 & 144.14 \\
6 & -14.30 & -0.51 \\
TS & -12.43 & -67.63 \\
9 & 128.86 & 190.54 \\
11 & 81.97 & 113.45 \\
\hline
\end{tabular}

\subsection{Stability of Reactive Intermediates}

Since the reaction of interest is the nucleophilic attack of water on the TAML system, the reactive intermediate is required to be electrophilic. Most stable spin states of each step in the mechanism were previously determined. It was found that 6 is a neutral triplet structure where the TAML ring was oxidised. $[32,46]$

Visualisation of the molecular orbitals (MOs) shows that for this open shell system we obtain two singly occupied molecular orbitals (SOMOs). Figure 4.1(a) and (b) reveals an $\alpha$-SOMO involving linear combinations of 3d (Fe), 
$2 p(N)$ and $2 p(O)$ orbitals and significant contributions from the aromatic $\pi$ system of the TAML ring on the $\beta$-SOMO. This indicates that the TAML ring is non-innocent. [38] This appears to be consistent for $6 \mathrm{~A}, 6 \mathrm{~B}, 6 \mathrm{C}$ and $6 \mathrm{D}$ (Figure A1).

Figure 4.1(c) and (d) present a different result. The SOMOs have a bonding combination for the Fe-O bond which may impede a nucleophile (usually attacks LUMO) from interacting with the supposed electrophilic Fe-O core of the reactive intermediate. In fact, modelling the TS for substitution pattern E was unsuccessful and further discussion on the matter proceeds below.

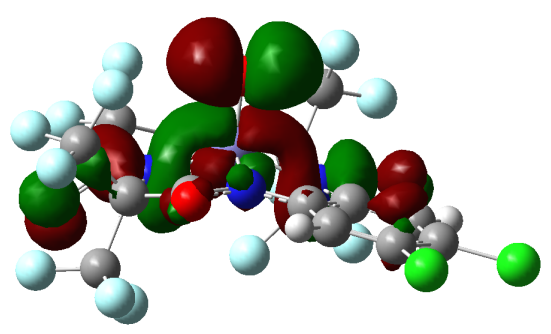

(a) $\alpha$-SOMO 6A

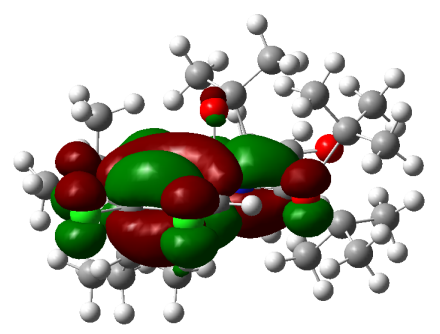

(c) $\alpha$-SOMO 6E

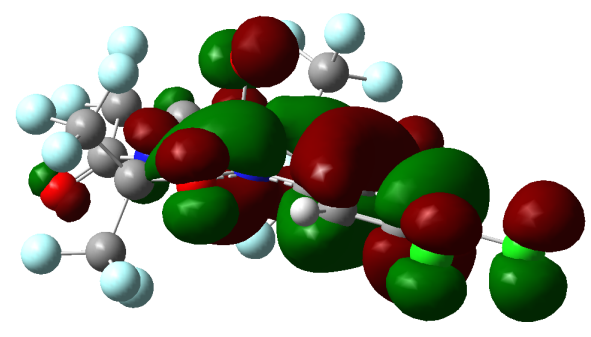

(b) $\beta$-SOMO 6A

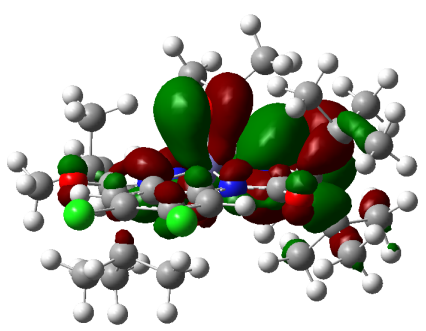

(d) $\beta$-SOMO 6E

Figure 4.1: MO contour plots of singly occupied molecular orbitals (SOMOs) for reactive intermediate structure at PBE0/Def2-TZVP level of theory. Molecules rotated to give clarity for the orbital contours. 


\subsection{Limiting Factors of Ligand Design}

Multiple attempts were made to model the water nucleophilic attack TS on structure E. In an effort to account for the steric bulk of the tert-butyl and iso-propyl groups on the TAML ring, the local solvation shell was reoriented but to no avail.

Initially, it was suspected that steric hinderance was the major contributing factor the the failed nucleophilic attack. However, the space-filling model (Figure 4.2) suggests a more subtle factor. The water molecules come in close proximity but not within van der Waals radii of the carbon groups (there is a degree of repulsion). More notably the branched ligands seems to push with the solvation shell in such that the a hydrogen in the water molecule at the back of the system is now interacting with the core of the TAML system. This behaviour further highlights the non-innocence of the TAML ring system in the reactive intermediate.

Moreover, similar occurrence is observed for the TS of structure C. This leads us to propose the iso-propyl ends on the TAML ring inhibit the nucleophilic attack.

NBO analysis indicates that for these structures there is a considerably higher Wiberg bond index value (Table 4.2) for the hydrogen - Fe-oxo core interaction compared to with the desired water (nucleophile) - Fe-oxo core interaction. The successful nucleophilic reactions have Wiberg bond indices that are of different magnitudes for the different interactions. Whereas, the proposed inhibited reactions have comparable Wiberg bond indices. 


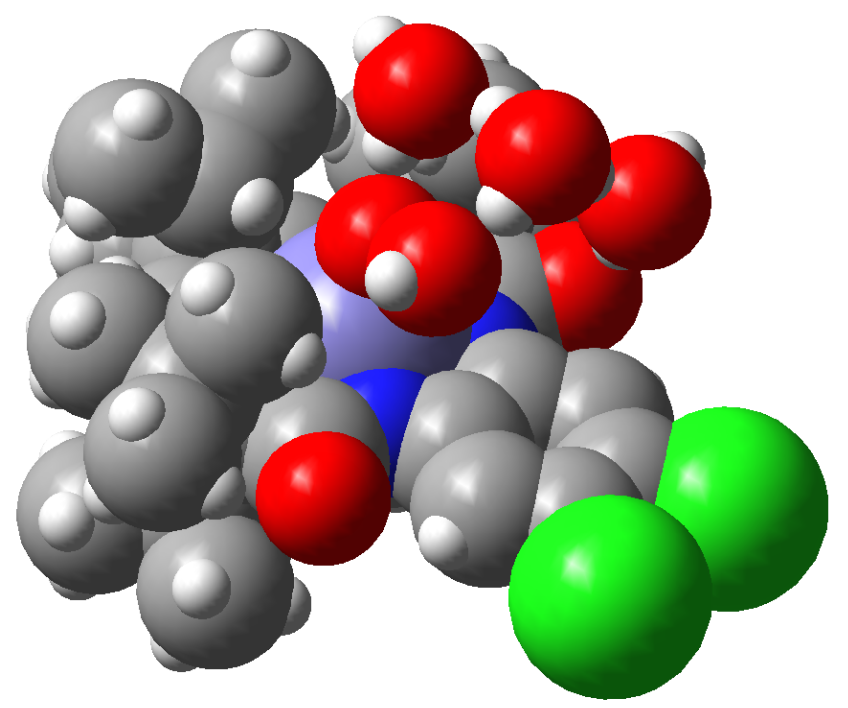

(a) Structure along optimisation of TS E with imaginary frequency

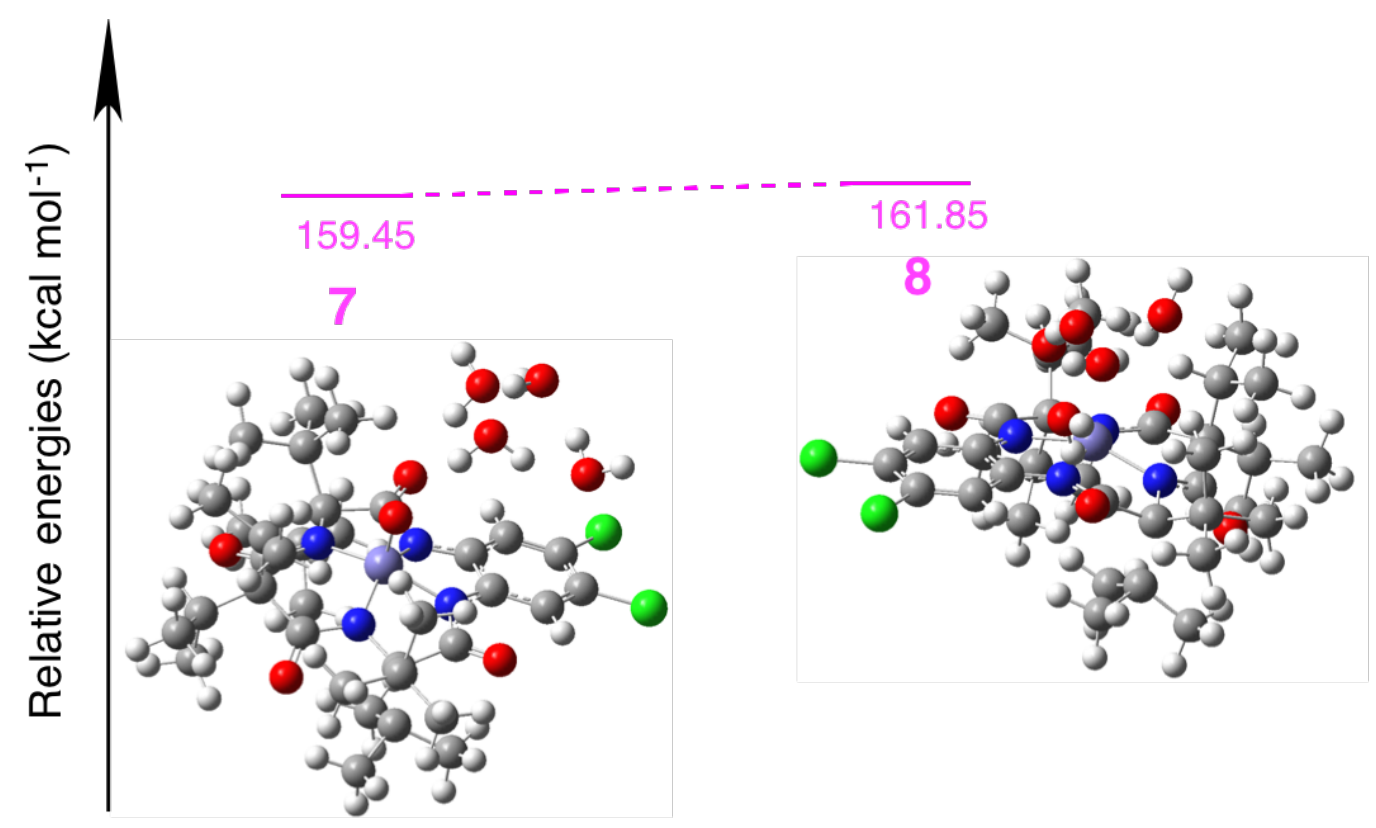

(b) Structures 7 and 8

Figure 4.2: Space-filling model of a structure along the optimisation of TS E that had an imaginary frequency which did not correspond the nucleophilic attack. Structures $\mathbf{7}$ and $\mathbf{8}$ provide insight that the solvation shell is not likely to facilitate the desired reaction. 
Table 4.2: Wiberg bond indices from NBO calculation at PBE0/Def2-TZVP level of theory.

\begin{tabular}{ccc}
\hline & H-Fe-oxo & Nuc-Fe-oxo \\
\hline B & 0.0038 & 0.5298 \\
C & 0.0386 & 0.0545 \\
D & 0.1273 & 0.9900 \\
E & 0.0097 & 0.0001 \\
\hline
\end{tabular}

\subsection{Thermodynamics of Reactions}

The focus of this work was to assess the effect of the TAML ring substitution pattern on the reactivity for the nucleophilic attack step of the fast water oxidation mechanism. Herein, the $\Delta \mathrm{G}$ values (Table 4.3) indicate full EWG substitution (B) decreases the activation barrier by $\sim 7 \mathrm{kcal} \mathrm{mol}^{-1}$ from the experimental structure (A). Moreover, D (same substitution pattern as (A) with tert-butyl groups instead of methyl groups) decreases the activation barrier by $\sim 8 \mathrm{kcal} \mathrm{mol}^{-1}$ from the experimental structure (A).

Perhaps more interestingly, $\mathbf{C}$ did not decrease the activation barrier. Upon closer inspection of the TS, the desired nucleophilic attack had not occurred due factors discussed earlier. The iso-propyl group had pushed the water molecules into a similar orientation. An interaction between the hydrogen and Fe-oxo core is proposed to impede the proton relay required for the nucleophilic attack (Figure 3.7). Thus, the $\Delta \mathrm{H}$ and $\Delta \mathrm{G}$ values obtained for the TS on structure $\mathbf{C}$ correspond to a different reaction coordinate. 
From this we can propose that the fluorinated end of the TAML structure was imperative for the local solvation shell to remain as a proton relay for the nucleophilic attack to occur. Conversely, iso-propyl ends of the TAML structure inhibit the desired nucleophilic attack of water on the reactive intermediate.

Table 4.3: Relative Enthalpies and Gibb's free energies for structures TS A, TS B, TS C and TS D. Calculated at PBE0/Def2-TZVP level of theory. All energies in kcal $\mathrm{mol}^{-1}$.

\begin{tabular}{lrr}
\hline & \multicolumn{1}{c}{$\Delta \mathrm{H}$} & \multicolumn{1}{c}{$\Delta \mathrm{G}$} \\
\hline $\mathrm{A}$ & 11.07 & 16.61 \\
$\mathrm{~B}$ & 3.40 & 9.12 \\
$\mathrm{C}$ & 14.28 & 17.09 \\
$\mathrm{D}$ & 0.63 & 7.94 \\
\hline
\end{tabular}




\section{Chapter 5}

\section{Conclusion}

With the advent of iron-TAML activators in the area of renewable energy production, a viable technology was developed to catalyse fast water oxidation. [31] This was studied mechanistically to establish the reaction mechanism by which this proceeds and provide a computational approach to further understand the catalyst design. [32, 46]

This present study investigated the iron-TAML catalysed fast water oxidation mechanism using DFT methods. The purpose was to quantitively model the proposed TS (nucleophilic attack of water) from earlier work. [32] This was performed on a variety of substitution arrangements on the TAML ring as to ascertain the effects of the ligands for the stabilisation of intermediates and activation energies.

It was found that the substitution patterns investigated yielded no apparent trend for the stabilisation of the PCET steps or the activation energies. However, the importance of the fluorinated TAML ends was highlighted by the impeded nucleophilic attack on structures lacking the fluorine ends (structures containing excess of steric repulsion). This had serious implications on the orientation of the solvation shell required for the production of the ferryl peroxide species. 
Furthermore, examination of the SOMOs for the reactive intermediates indicated the substitution rearrangement did not significantly alter the orbital contributions of the TAML ring to the MOs in comparison to the experimental structure. Hence, the TAML species proposed in this study should be considered non-innocent and thus, not able to stabilise the intermediate structure. $[38,32]$ Notably, the orbital contributions for the aformentioned SOMOs primarily comes from the $\pi$ system of the TAML ring. Perhaps, modification of the $\pi$ system of the TAML structure may prove a useful next step for mechanistic studies.

A definite conclusion that can be drawn is that, compared to the experimental structure A, a mixture of EDGs and EWGs with a fluorinated end on the TAML structure (D) can halve the activation energy. Additionally, to avoid the competing interactions of the solvation shell, TAML structure (B) is predicted to lower the activation energy by $7 \mathrm{kcal} \mathrm{mol}^{-1}$ compared to experimental structure A. Therefore, we propose either structure B or D as viable candidates for experimental study. 


\section{Appendix A}

Table A1: Sum of electronic and zero-point energies in a.u. calculated at PBE0/Def2-TZVP level of theory.

\begin{tabular}{lccccc}
\hline & $\mathrm{A}$ & $\mathrm{B}$ & $\mathrm{C}$ & $\mathrm{D}$ & $\mathrm{E}$ \\
\hline 1 & -3634.799645 & -4825.243032 & -4862.307658 & -4105.703786 & -4142.749629 \\
2 & -3634.643165 & -4825.064646 & -4862.146633 & -4105.545552 & -4142.570092 \\
3 & -3634.165083 & -4824.598665 & -4861.678266 & -4105.066295 & -4142.126302 \\
4 & -3633.981109 & -4824.409083 & -4861.496845 & -4104.899620 & -4141.964418 \\
5 & -3633.513592 & -4823.943088 & -4861.026920 & -4104.413678 & -4141.478251 \\
6 & -3633.342329 & -4823.749777 & -4860.829002 & -4104.244294 & -4141.301697 \\
7 & -3938.805980 & -5129.213709 & -5166.304858 & -4409.705169 & -4446.776280 \\
TS & -3938.785378 & -5129.205269 & -5166.281031 & -4409.700405 & \\
8 & -3938.812468 & -5129.235137 & -5166.318330 & -4409.709599 & -4446.772502 \\
9 & -3709.248349 & -4899.687342 & -4936.763704 & -4180.148565 & -4217.210707 \\
10 & -3709.059858 & -4899.505738 & -4936.585347 & -4179.989746 & -4217.046370 \\
11 & -3708.659425 & -4899.098830 & -4936.178283 & -4179.565102 & -4216.623787 \\
\hline
\end{tabular}


Table A2: Sum of electronic and thermal energies in a.u. calculated at PBE0/Def2TZVP level of theory.

\begin{tabular}{lccccc}
\hline & $\mathrm{A}$ & $\mathrm{B}$ & $\mathrm{C}$ & $\mathrm{D}$ & $\mathrm{E}$ \\
\hline 1 & -3634.769529 & -4825.204715 & -4862.262457 & -4105.659015 & -4142.697379 \\
2 & -3634.613018 & -4825.026227 & -4862.101808 & -4105.500614 & -4142.518737 \\
3 & -3634.135998 & -4824.561325 & -4861.634453 & -4105.022404 & -4142.075924 \\
4 & -3633.951861 & -4824.371670 & -4861.452971 & -4104.855539 & -4141.913125 \\
5 & -3633.485257 & -4823.906583 & -4860.983911 & -4104.370846 & -4141.428811 \\
6 & -3633.313876 & -4823.713102 & -4860.785242 & -4104.201135 & -4141.250972 \\
7 & -3938.764888 & -5129.164181 & -5166.248918 & -4409.649194 & -4446.714971 \\
$\mathrm{TS}$ & -3938.747250 & -5129.158764 & -5166.226161 & -4409.648188 & \\
8 & -3938.774441 & -5129.188585 & -5166.265437 & -4409.657070 & -4446.713672 \\
9 & -3709.217837 & -4899.648575 & -4936.718362 & -4180.103426 & -4217.159017 \\
10 & -3709.029429 & -4899.466651 & -4936.539724 & -4179.943812 & -4216.994977 \\
11 & -3708.627886 & -4899.059169 & -4936.132023 & -4179.518539 & -4216.570715 \\
\hline
\end{tabular}

Table A3: Sum of electronic and thermal free energies in a.u. calculated at PBE0/Def2-TZVP level of theory.

\begin{tabular}{lccccc}
\hline & $\mathrm{A}$ & $\mathrm{B}$ & $\mathrm{C}$ & $\mathrm{D}$ & $\mathrm{E}$ \\
\hline 1 & -3634.859973 & -4825.314815 & -4862.386212 & -4105.776152 & -4142.827824 \\
2 & -3634.703394 & -4825.136648 & -4862.223689 & -4105.618424 & -4142.647788 \\
3 & -3634.224236 & -4824.669227 & -4861.754234 & -4105.137743 & -4142.203397 \\
4 & -3634.040378 & -4824.479607 & -4861.572775 & -4104.971599 & -4142.041918 \\
5 & -3633.571772 & -4824.012775 & -4861.102093 & -4104.483502 & -4141.553825 \\
6 & -3633.401191 & -4823.820326 & -4860.906040 & -4104.315224 & -4141.379187 \\
7 & -3938.881721 & -5129.301402 & -5166.397945 & -4409.792890 & -4446.868450 \\
TS & -3938.855250 & -5129.286863 & -5166.370709 & -4409.780243 & \\
8 & -3938.882661 & -5129.317633 & -5166.405934 & -4409.790985 & -4446.858598 \\
9 & -3709.309114 & -4899.759622 & -4936.841677 & -4180.221778 & -4217.289565 \\
10 & -3709.120780 & -4899.578970 & -4936.664614 & -4180.064365 & -4217.123485 \\
11 & -3708.724325 & -4899.174956 & -4936.260419 & -4179.644629 & -4216.709289 \\
\hline
\end{tabular}




\section{Appendix B}

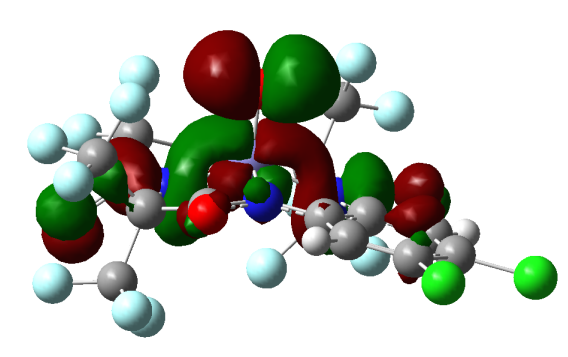

(a) $\alpha$-SOMO 6B

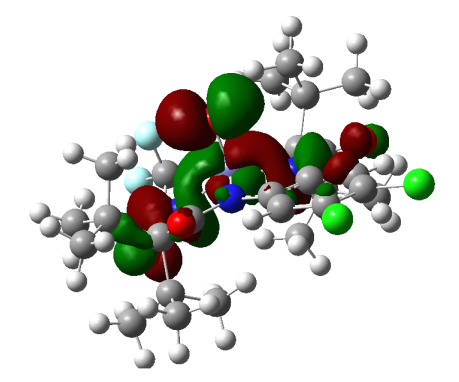

(c) $\alpha$-SOMO 6D

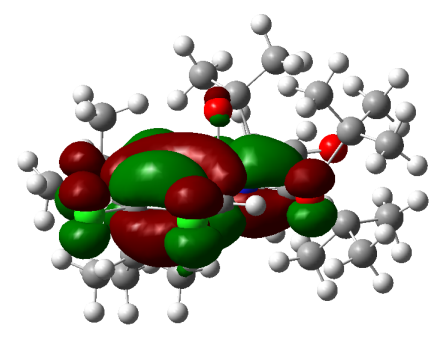

(e) $\alpha$-SOMO 6E

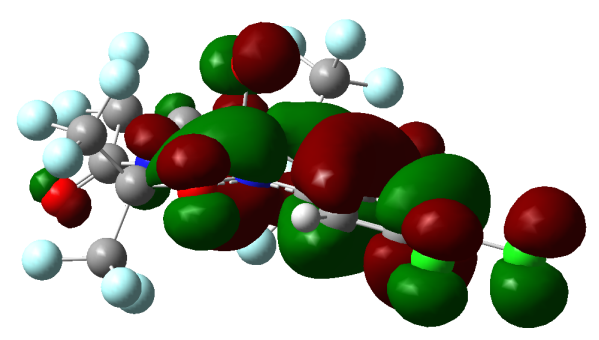

(b) $\beta$-SOMO 6B

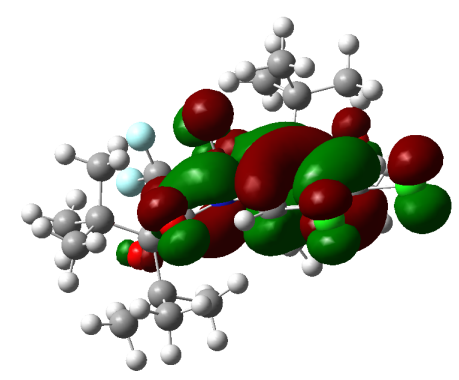

(d) $\beta$-SOMO 6D

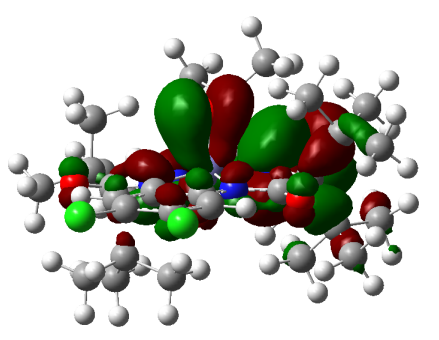

(f) $\beta$-SOMO 6E

Figure B1: MO contour plots of singly occupied molecular orbitals (SOMOs) for reactive intermediate structure at PBE0/Def2-TZVP level of theory. Molecules rotated to give clarity for the orbital contours. 


\section{Appendix C}

This appendix contains the full reaction mechanisms for all iron-TAML systems presented in this study. All calculations were performed at PBE0/Def2TZVP level of theory. Energies given are the electronic energies with zeropoint energy corrections in $\mathrm{kcal} \mathrm{mol}^{-1}$. PCET steps are corrected by the free energy of an electron ( $\mathrm{Li} / \mathrm{Li}^{+}$redox couple) was calculated to be -128.42 $\mathrm{kcal} \mathrm{mol}^{-1}$ at PBE0/Def2-TZVP and the free energy of a proton calculated at the same level of theory to be $-165.82 \mathrm{kcal} \mathrm{mol}^{-1}$. Solvation shell was also calculated at same level of theory. 

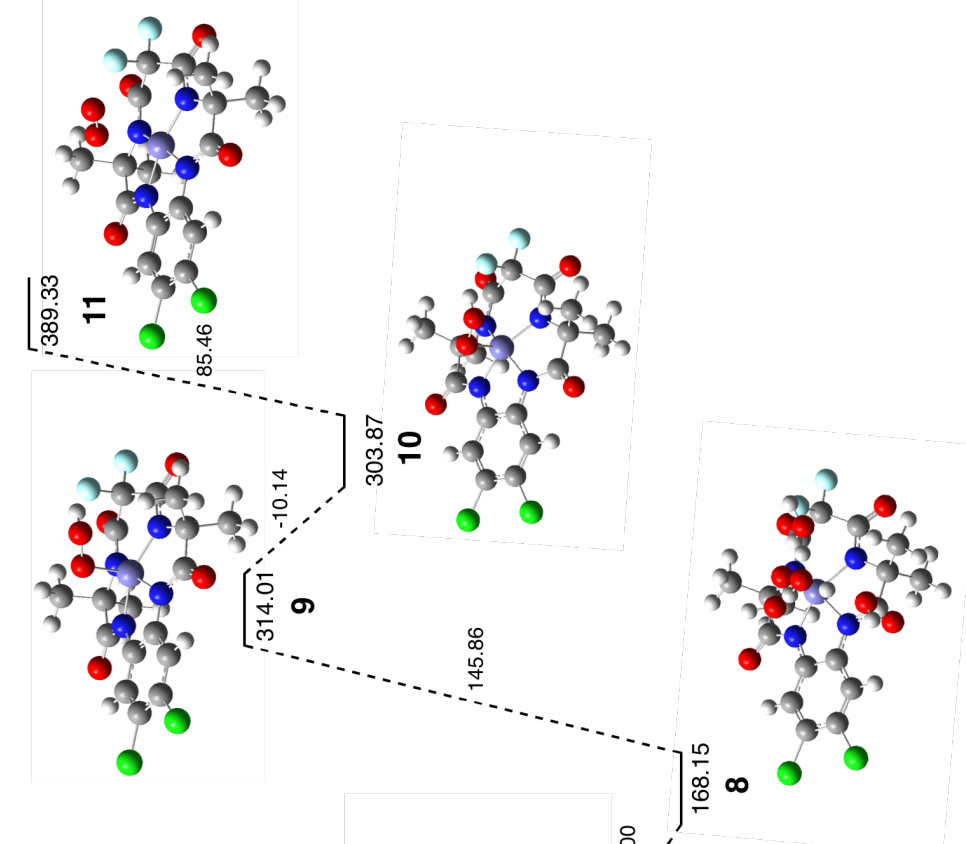

总

. ธี

के

ธี

疍.

.

1

$\stackrel{\mathscr{I}}{\square}$

.

离

क षี

0

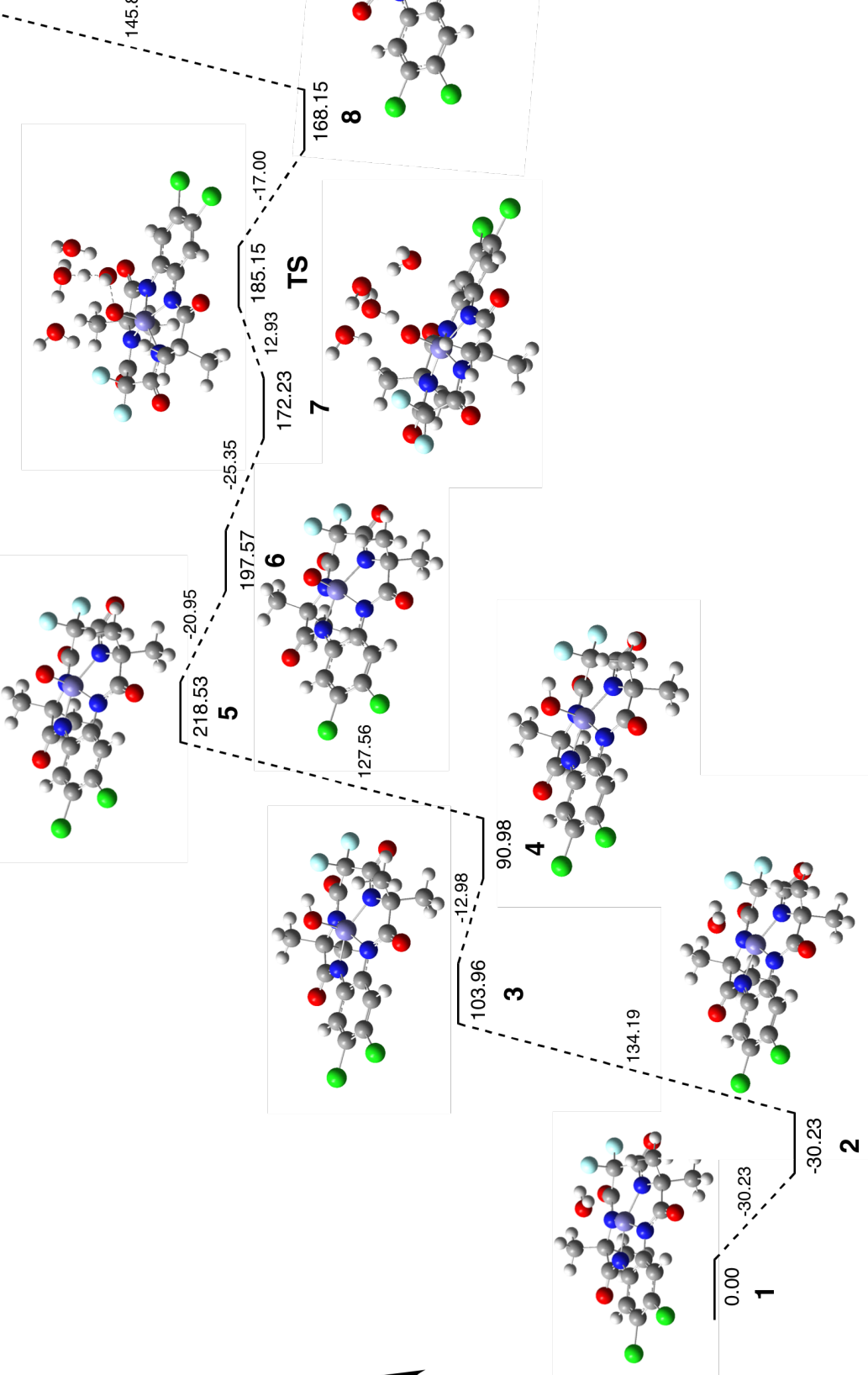

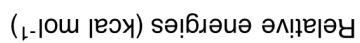

击

$\wedge \quad \overrightarrow{0}$

\& 


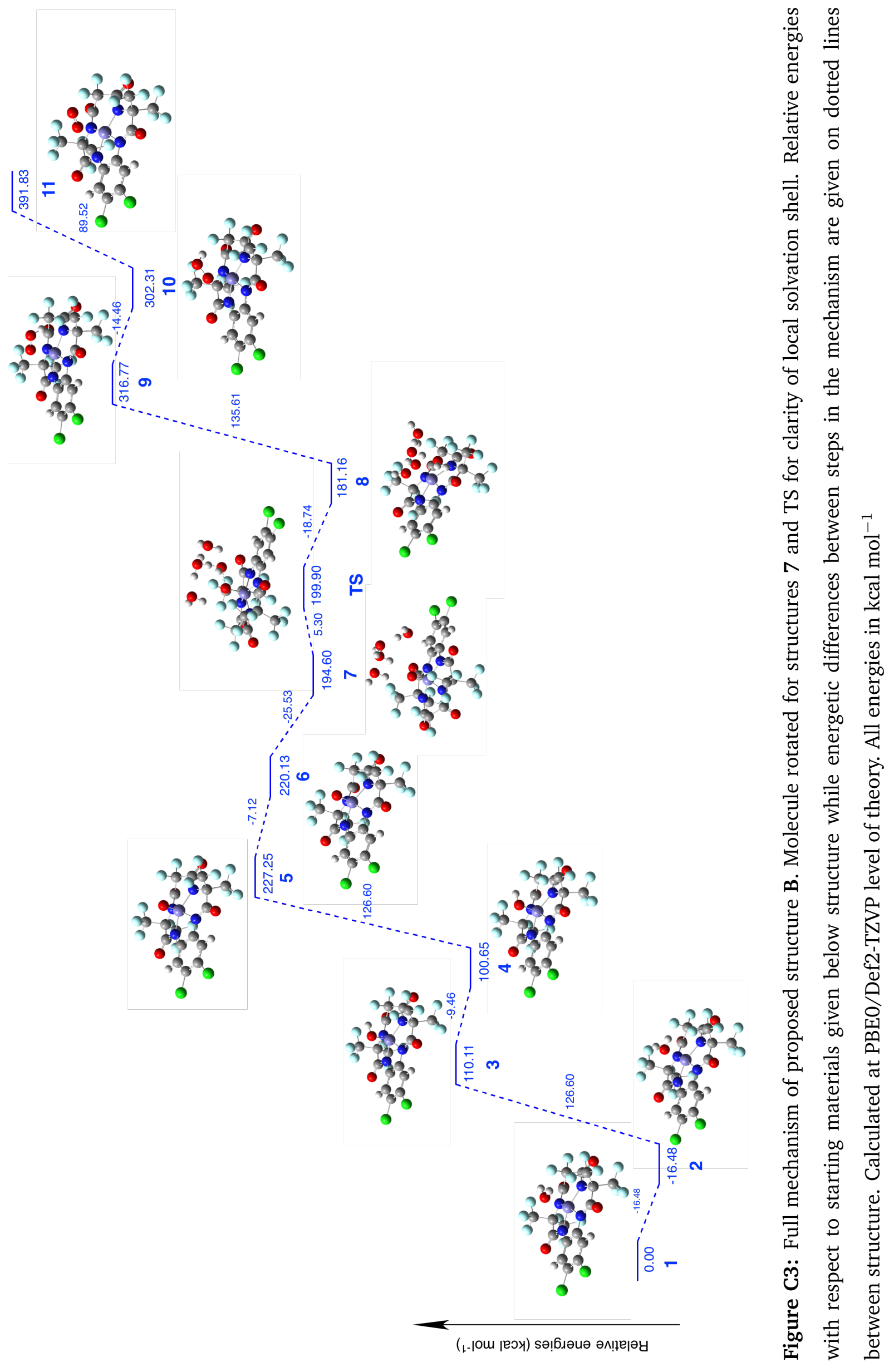



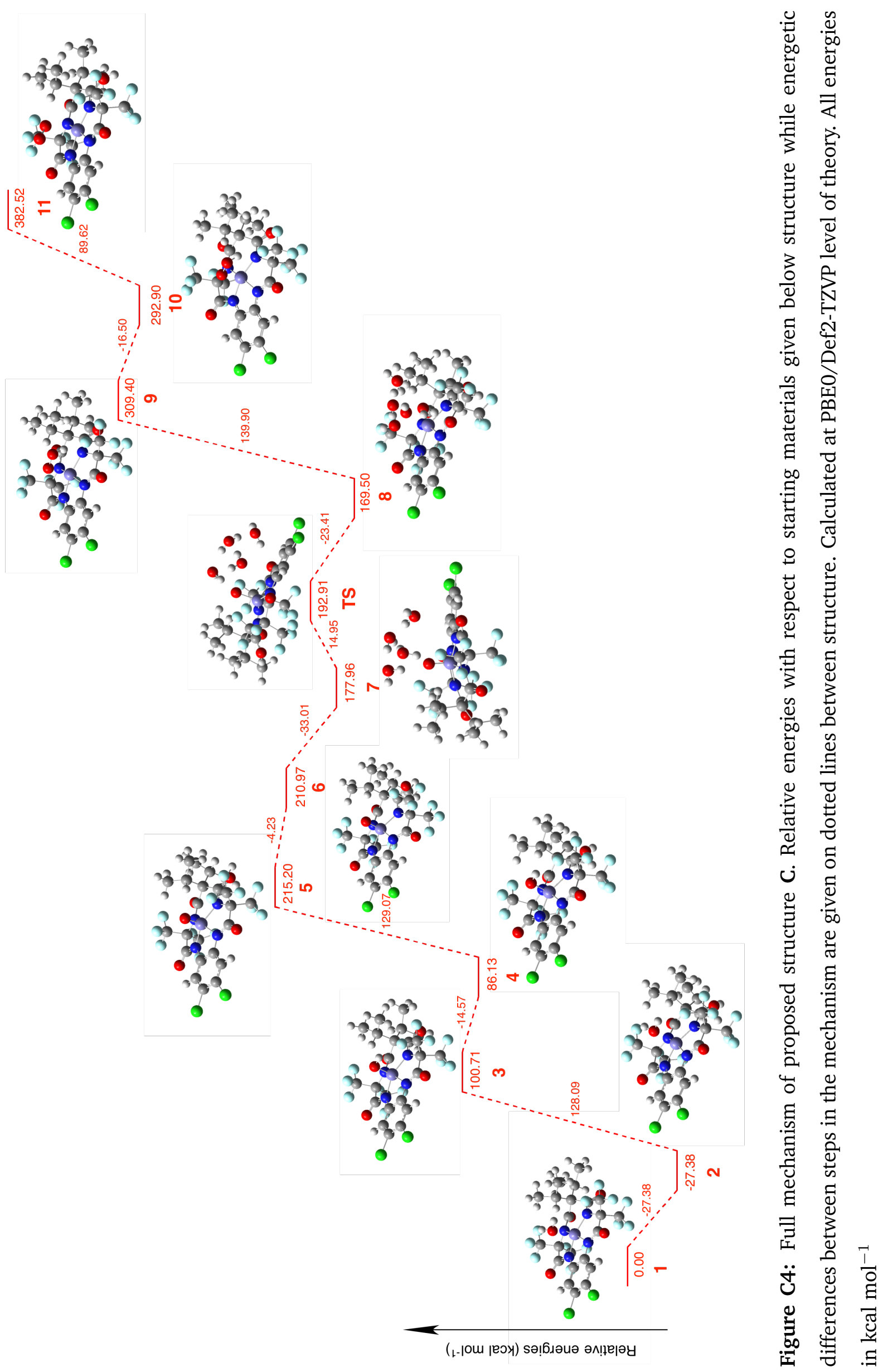


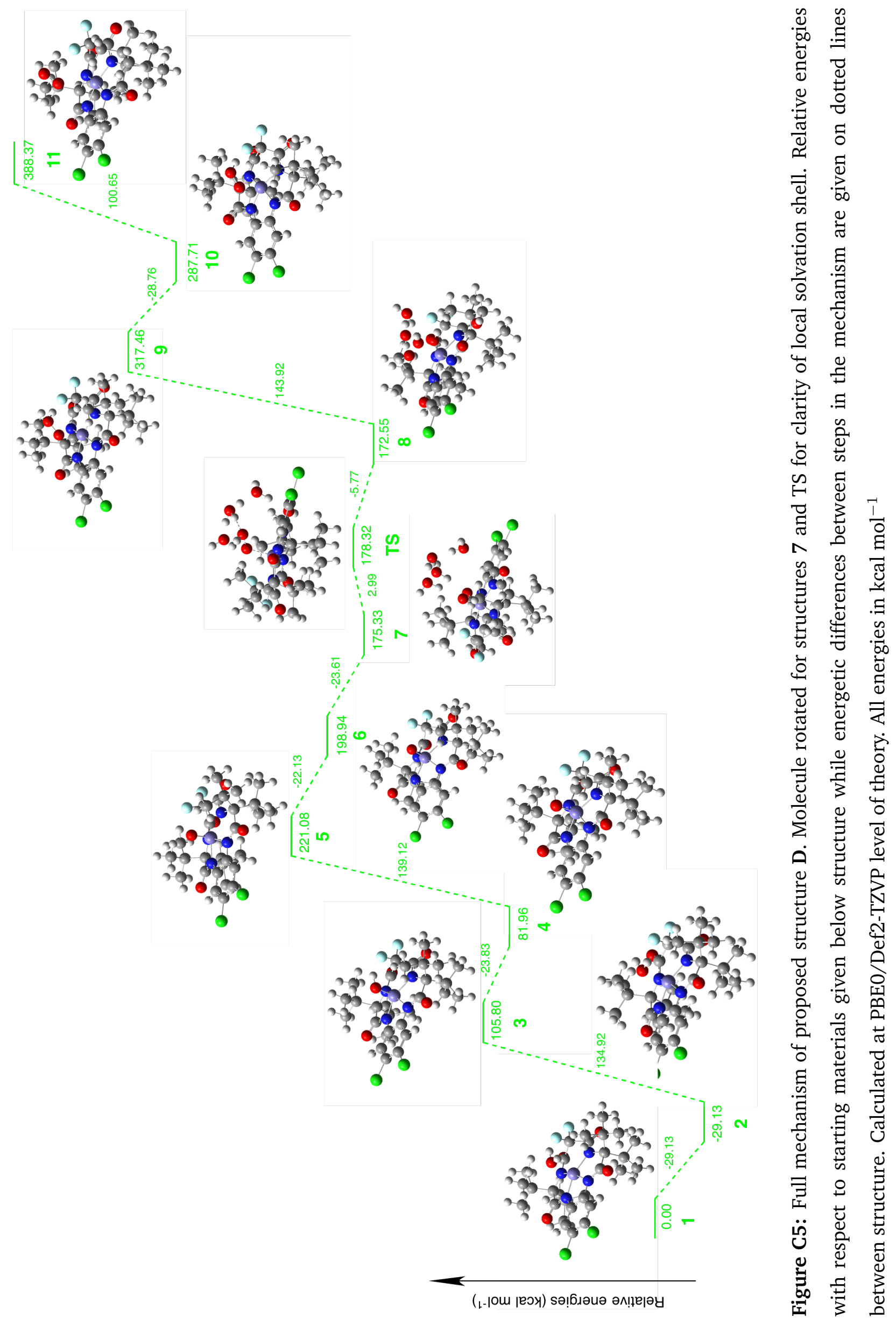




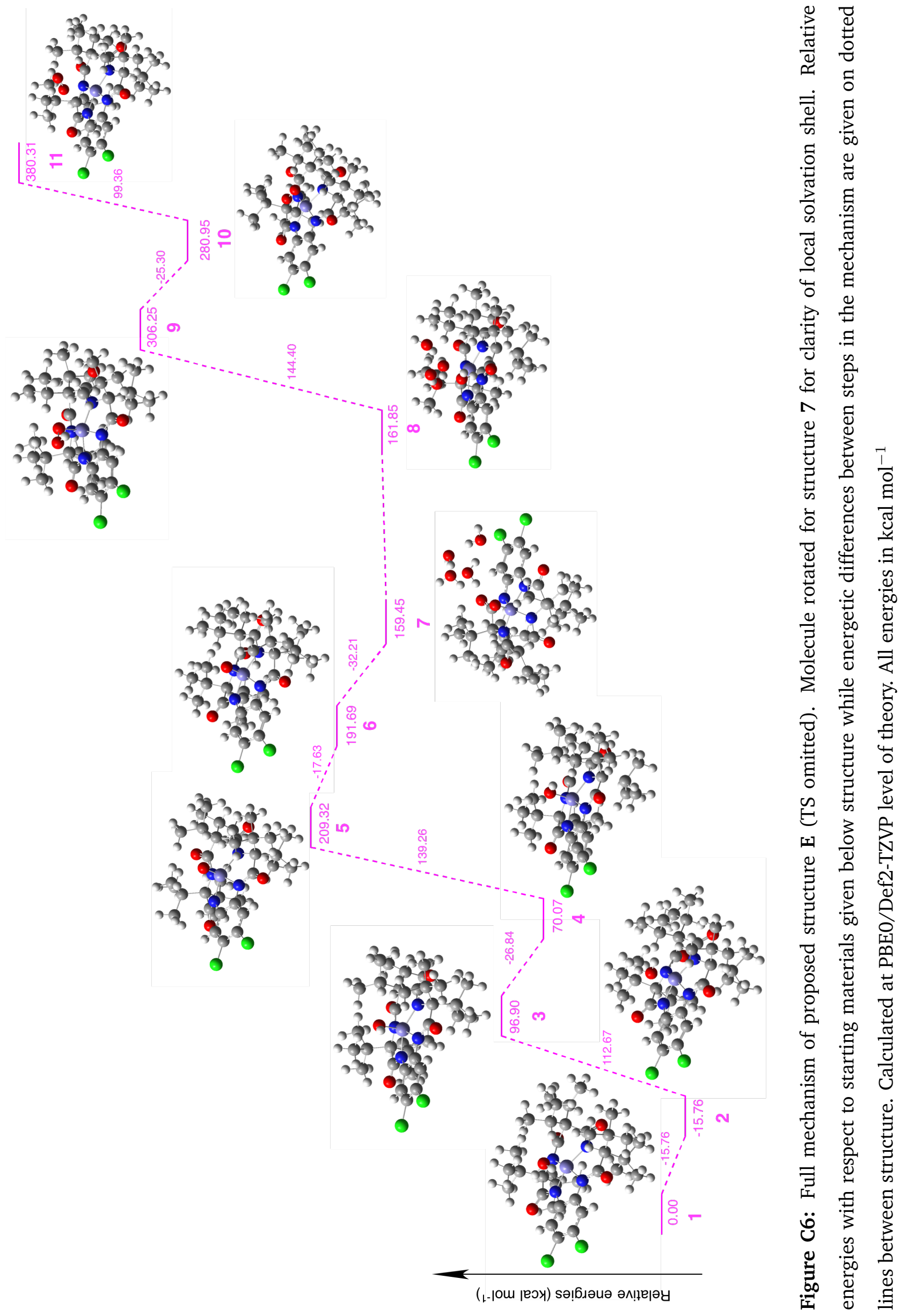




\section{Bibliography}

[1] T. J. Collins; C. Walter. "Little green molecules". In: Scientific American 294.3 (2006), pp. 82-90.

[2] T. J. Collins. "TAML oxidant activators: A new approach to the activation of hydrogen peroxide for environmentally significant problems". In: Accounts of Chemical Research 35.9 (2002), pp. 782-790.

[3] A. Ghosh; S. S. Gupta; M. J. Bartos; Y. Hangun; L. D. Vuocolo.; B. A. Steinhoff; C. A. Noser; D. Horner; S. Mayer; K. Inderhees; C. P Horwitz; J. Spatz; A. D. Ryabov.; S. Mondal; T. J. Collins. "Green chemistry. Sustaining a high-technology civilization". In: Pure and Applied Chemistry 73.1 (2001), pp. 113-118.

[4] T. J. Collins. "Designing ligands for oxidizing complexes". In: Accounts of Chemical Research 27.9 (1994), pp. 279-285.

[5] Brewer J. C.; T. J. Collins; Smith M. R.; Santarsiero B. D. "Neutral square planar cobalt(III) complexes". In: Journal of the American Chemical Society 110.2 (1988), pp. 423-428.

[6] V. J. Brown. "Fe-TAML catalyst for cleanup". In: Environmental Health Perspectives 114.11 (2006), pp. 656-659.

[7] A. Chanda; A. D. Ryabov.; S. Mondal; L. Alexandrova; A. Ghosh; Y. HangunBalkir; C. P Horwitz; T. J. Collins. "Activity-stability parameterization of homogeneous green oxidation catalysts". In: Chemistry - A European Journal 12.36 (2006), pp. 9336-9345. 
[8] W. C. Ellis; C. T. Tran; M. A. Denardo; A. Fischer; A. D. Ryabov; T. J. Collins. "Design of more powerful iron-TAML peroxidase enzyme mimics". In: Journal of the American Chemical Society 131.50 (2009), pp. 18052-18053.

[9] C. W. Jones. "Applications of Hydrogen Peroxide; Derivatives". In: RSC Clean Technology Monographs (1999). Ed. by The Royal Society of Chemistry.

[10] T. J. Collins; S. W. Gordon-Wylie. "A manganese(V)-oxo complex". In: Journal of the American Chemical Society 111.12 (1989), pp. 45114513.

[11] T. J. Collins; K. L. Kostka; E. S. Uffelman; T. L. Weinberger. "Design synthesis and structure of a macrocyclic tetraamide that stabilizes high-valent middle and later transition metals". In: Inorganic Chemistry 30.22 (1991), pp. 4204-4210.

[12] T. J. Collins; R. D. Powell; C. Slebodnick; E. S. Uffelman. "Stable highly oxidizing cobalt complexes of macrocyclic ligands". In: Journal of the American Chemical Society 113.22 (1991), pp. 8419-8425.

[13] T. J. Collins; B. G. Fox; R. D. Powell; Z. G. Hu; K. L. Kostka; E. Münck; C. E. F. Rickard; L. J. Wright. "High valent transition metal chemistry. Synthesis and characterization of an intermediate-spin iron(IV) complex of a strong $\pi$-acid ligand". In: Journal of the American Chemical Society 114.22 (1992), pp. 8724-8725.

[14] M. J. Bartos; S. W. Gordon-Wylie; B. G. Fox; L. J. Wright; S. T. Weintraub; K. E. Kauffmann; E. Münck; K. L. Kostka; E. S. Uffelman; C. E. F. Rickard; K. R. Noon; T. J. Collins. "Designing ligands to achieve robust oxidation catalysts. Iron based systems". In: Coordination Chemistry Reviews 174.1 (1998), pp. 361-390.

[15] V. Polshin; D. L. Popescu; A. Fischer; A. Chanda; D. D. Horner; E. S. Beach;

J. Henry; Y. L. Qian; C. P Horwitz; G. Lente; I. Fabian; E. Münck; E. L. Bominaar; A. D. Ryabov.; T. J. Collins. "Attaining control by de- 
sign over the hydrolytic stability of Fe-TAML oxidation catalysts". In: Journal of the American Chemical Society 130.13 (2008), pp. 44974506.

[16] J. A. Miller; L. Alexander; D. I. Mori; A. D. Ryabov.; T. J. Collins. "In situ enzymatic generation of $\mathrm{H} 2 \mathrm{O} 2$ from $\mathrm{O} 2$ for use in oxidative bleaching and catalysis by TAML activators". In: New Journal of Chemistry 37.11 (2013), pp. 3488-3495.

[17] W. C. Ellis; C. T. Tran; R. Roy; M. Rusten; A. Fischer; A. D. Ryabov.; B. Blumberg; T. J. Collins. "Designing green oxidation catalysts for purifying environmental waters". In: Journal of the American Chemical Society 132.28 (2010), pp. 9774-9781.

[18] S. Sen Gupta; M. Stadler; C. A. Noser; A. Ghosh; B. Steinhoff; D. Lenoir; C. P Horwitz; K. W. Schramm; T. J. Collins. "Rapid total destruction of chlorophenols by activated hydrogen peroxide". In: Science 296.5566 (2002), pp. 326-328.

[19] J. Raloff. “Pesticide Disposal Goes Green”. In: Science News (2004).

[20] N. Chahbane; D. Lenoir; S. Souabi; T. J. Collins; K. W. Schramm. "FeIII-TAML-Catalyzed green oxidative decolorization of textile dyes in wastewater". In: Clean - Soil Air Water 35.5 (2007), pp. 459-464.

[21] N. Chahbane; D. L. Popescu; D. A. Mitchell; A. Chanda; D. Lenoir; A. D. Ryabov.; K. W. Schramm; T. J. Collins. "FeIII-TAML-catalyzed green oxidative degradation of the azo dye Orange II by H2O2; organic peroxides: Products toxicity kinetics and mechanisms". In: Green Chemistry 9.1 (2007), pp. 49-57.

[22] E. S. Beach; R. T. Malecky; R. R. Gil; C. P Horwitz; T. J. Collins. "Fe-TAML/hydrogen peroxide degradation of concentrated solutions of the commercial azo dye tartrazine". In: Catalysis Science and Technology 1.3 (2011), pp. 437-443. 
[23] S. Kundu; A. Chanda; S. K. Khetan; A. D. Ryabov.; T. J. Collins. "TAML activator/peroxide-catalyzed facile oxidative degradation of the persistent explosives trinitrotoluene and trinitrobenzene in micellar solutions". In: Environmental Science and Technology 47.10 (2013), pp. 53195326.

[24] S. Kundu; A. Chanda; J. V. K. Thompson; G. Diabes; S. K. Khetan; A. D. Ryabov; T. J. Collins. "Rapid degradation of oxidation resistant nitrophenols by TAML activator; H2O2". In: Catalyst Science; Technology 5.3 (2015), pp. 1775-1782.

[25] M. Paul; B. Setlow; P. Setlow. "Killing of spores of Bacillus subtilis by tert-butyl hydroperoxide plus a TAML(C) activator". In: Journal of Applied Microbiology 102.4 (2007), pp. 954-962.

[26] D. Banerjee; A. L. Markley; T. Yano; A. Ghosh; P. B. Berget; E. G. Minkley; S. K. Khetan; T. J. Collins. "Green Oxidation Catalysis for Rapid Deactivation of Bacterial Spores". In: Angewandte Chemie International Edition 45.24 (2006), pp. 3974-3977.

[27] S. Mondal; Y. Hangun-Balkir; L. Alexandrova; D. Link; B. Howard; P. Zandhuis; A. Cugini; C. P Horwitz; T. J. Collins. "Oxidation of sulfur components in diesel fuel using Fe-TAML(C) catalysts; hydrogen peroxide". In: Catalysis Today 116.4 (2006), pp. 554-561.

[28] A. Chanda; S. K. Khetan; D. Banerjee; A. Ghosh; T. J. Collins. "Total degradation of fenitrothion and other organophosphorus pesticides by catalytic oxidation employing Fe-TAML peroxide activators". In: Journal of the American Chemical Society 128.37 (2006), pp. 1205812059.

[29] L. Q. Shen; E. S. Beach; Y. Xiang; D. J. Tshudy; N. Khanina; C. P Horwitz; M. E. Bier; T. J. Collins. "Rapid biomimetic degradation in water of the persistent drug sertraline by TAML catalysts; hydrogen peroxide". In: Environmental Science; Technology 45.18 (2011), pp. 78827887. 
[30] M. R. Millis; K. Arias-Salazar; A. Baynes; L. Q. Shen; J. Churchley: N. Beresford; C. Gayathri; R. R. Gil; R. Kanda; S. Jobling; T. J. Collins. "Removal of ecotoxicity of $17 \alpha$-ethinylestradiol using TAML/peroxide water treatment". In: Scientific Reports 5 (2015), p. 10511.

[31] W. C. Ellis; N. D. McDaniel; S. Bernhard; T. J. Collins. "Fast water oxidation using Iron". In: Journal of the American Chemical Society 132.32 (2010), pp. 10990-10991.

[32] M. Z. Ertem; L. Gagliardi; C. J. Cramer. "Quantum chemical characterization of the mechanism of an iron-based water oxidation catalyst". In: Chemical Science 3.4 (2012), pp. 1293-1299.

[33] A. Chanda; D. L. Popescu; F. T. De Oliveira; E. L. Bominaar; A. D. Ryabov.; E. Münck; T. J. Collins. "High-valent iron complexes with tetraamido macrocyclic ligands: Structures Mössbauer spectroscopy and DFT calculations". In: Journal of Inorganic Biochemistry 100.4 (2006), pp. 606619.

[34] F. T. De Oliveira; A. Chanda; D. Banerjee; X. Shan; S. Mondal; L. Que Jr; E. L. Bominaar; E. Münck; T. J. Collins. "Chemical; spectroscopic evidence for an FeV-oxo complex". In: Science 315.5813 (2007), pp. 835838.

[35] A. Chanda; X. Shan; M. Chakrabarti; W. C. Ellis; D. L. Popescu; F. T. De Oliveira; D. Wang; L. Que Jr; T. J. Collins; E. Münck; E. L. Bominaar. "(TAML)FeIV=O complex in aqueous solution: Synthesis and spectroscopic and computational characterization". In: Inorganic Chemistry 47.9 (2008), pp. 3669-3678.

[36] S. Kundu; M. Annavajhala; I. V. Kurnikov; A. D. Ryabov.; T. J. Collins. "Experimental and theoretical evidence for multiple FeIV reactive intermediates in TAML-activator catalysis: Rationalizing a counterintuitive reactivity order". In: Chemistry - A European Journal 18.33 (2012), pp. 10244-10249. 
[37] Van Heuvelen K. M.; A. T. Fiedler; X. Shan; R. F. De Hont; K. K. Meier; E. L. Bominaar; E. Münck; L. Que Jr. "One-electron oxidation of an oxoiron(IV) complex to form an $[\mathrm{O}=\mathrm{Fe} \mathrm{V}=\mathrm{NR}]+$ center". In: Proceedings of the National Academy of Sciences of the United States of America 109.30 (2012), pp. 11933-11938.

[38] M. M. Conradie; J. Conradie; A. Ghosh. "A DFT overview of highvalent iron cobalt and nickel tetraamidomacrocyclic ligand (TAML) complexes: The end of innocence?" In: Journal of Inorganic Biochemistry 100.4 (2006), pp. 620-626.

[39] J. P. Perdew; K. Burke; M. Ernzerhof. "Generalized gradient approximation made simple". In: Physical Review Letters 77.18 (1996), pp. 38653868.

[40] M. J. Frisch; G. W. Trucks; H. B. Schlegel; G. E. Scuseria; M. A. Robb; J. R. Cheeseman; G. Scalmani; V. Barone; B. Mennucci; G. A. Petersson; H. Nakatsuji; M. Caricato; X. Li; H. P. Hratchian; A. F. Izmaylov; J. Bloino; G. Zheng; J. L. Sonnenberg; M. Hada; M. Ehara; K. Toyota; R. Fukuda; J. Hasegawa; M. Ishida; T. Nakajima; Y. Honda; O. Kitao; H. Nakai; T. Vreven; J. A. Montgomery; Jr.; J. E. Peralta; F. Ogliaro; M. Bearpark; J. J. Heyd; E. Brothers; K. N. Kudin; V. N. Staroverov; R. Kobayashi; J. Normand; K. Raghavachari; A. Rendell; J. C. Burant; S. S. Iyengar; J. Tomasi; M. Cossi; N. Rega; J. M. Millam; M. Klene; J. E. Knox; J. B. Cross; V. Bakken; C. Adamo; J. Jaramillo; R. Gomperts; R. E. Stratmann; O. Yazyev; A. J. Austin; R. Cammi; C. Pomelli; J. W. Ochterski; R. L. Martin; K. Morokuma; V. G. Zakrzewski; G. A. Voth; P. Salvador; J. J. Dannenberg; S. Dapprich; A. D. Daniels; Ö. Farkas; J. B. Foresman; J. V. Ortiz; J. Cioslowski; D. J. Fox. Gaussian 09 Revision E.01. Ed. by Gaussian Inc. Wallingford CT. Miscellanous. 2009.

[41] J. P. Perdew; K. Burke; M. Ernzerhof. "Errata: Generalized gradient approximation made simple". In: Physical Review Letters 78.7 (1997), pp. 1396-1396. 
[42] C. Adamo; V. Barone. "Toward reliable density functional methods without adjustable parameters: The PBE0 model". In: Journal of Chemical Physics 110 (1999), pp. 6158-6169.

[43] F. Weigend; R. Ahlrichs. "Balanced basis sets of split valence triple zeta valence; quadruple zeta valence quality for $\mathrm{H}$ to $\mathrm{Rn}$ : Design and assessment of accuracy". In: Physical Chemistry Chemical Physics 7 (2005), pp. 3297-3305.

[44] F. Weigend. "Accurate Coulomb-fitting basis sets for H to Rn". In: Physical Chemistry Chemical Physics 8 (2006), pp. 1057-1065.

[45] E. D. Glendening; A. E. Reed; J. E. Carpenter; F. Weinhold. NBO Version E.01. Miscellanous.

[46] R. Z. Liao; X. C. Li; P. E. M. Siegbahn. "Reaction mechanism of water oxidation catalyzed by iron tetraamido macrocyclic ligand complexes - A DFT study". In: European Journal of Inorganic Chemistry 4 (2014), pp. 728-741. 